\title{
A Multi-Channel Scattering Theory for Some Time Dependent Hamiltonians, Charge Transfer Problem
}

\section{Kenji Yajima *}

Department of Mathematics, University of Tokyo, Tokyo 113, Japan

\begin{abstract}
Scattering theory for time dependent Hamiltonian $H(t)=$ $-(1 / 2) \Delta+\sum V_{j}\left(x-q_{j}(t)\right)$ is discussed. The existence, asymptotic orthogonality and the asymptotic completeness of the multi-channel wave operators are obtained under the conditions that the potentials are short range: $\left|V_{j}(x)\right| \leqq C_{j}(1+|x|)^{-2-\varepsilon}$, roughly spoken; and the trajectories $q_{j}(t)$ are straight lines at remote past and far future, and $\left|q_{j}(t)-q_{k}(t)\right| \rightarrow \infty$ as $t \rightarrow \pm \infty(j \neq k)$.
\end{abstract}

\section{Introduction}

The purpose of this paper is to study the scattering theory for a class of Schrödinger equations with time dependent potentials

$$
i \frac{\partial u}{\partial t}(t, x)=-\frac{1}{2} \Delta u(t, x)+\sum_{j=1}^{N} V_{j}\left(x-q_{j}(t)\right) u(t, x),
$$

where $q_{j}(t) \in \mathbb{R}^{n}(n \geqq 3)$ are the functions of $t \in \mathbb{R}^{1}$ which are straight lines at remote past and far future.

Suppose that $N$-centres of forces are traveling along the given trajectories $q_{j}(t)(j=1,2, \ldots, N)$ each of which acts on a quantum mechanical particle of mass 1 through the potential $V_{j}(x)$, then the Schrödinger equation for the particle is written as (1.1). If $\left|q_{j}(t)-q_{k}(t)\right| \rightarrow \infty$ as $|t| \rightarrow \infty$ sufficiently rapidly in conjunction with the rate of decay of the potentials, one would naturally expect that the behaviour of the particle in far future or remote past are classified into $(N+1)$-ways: (1) The particle behaves like a free particle; (2) the particle travels with one of the centres $q_{j}(t)$ forming a bound state around the centre $(j=1,2, \ldots, N)$. We shall prove in this paper that this is actually what is going on with the equation (1.1) under a suitable condition. In physics literature these centres of forces are usually supposed to be atoms and ions, and the particle to be the electron. In such case the scattering theory

* Partly supported by Sakkô-kai Foundation. 
for (1.1) is nothing but to study how the electron is transfered from some atom or ion to another. This is the reason why the problem is named "charge transfer problem".

Assumption I. (1) For any $j=1,2, \ldots, N, q_{j}(t)$ is a continuously differentiable function from $\mathbb{R}^{1}$ to $\mathbb{R}^{n}$.

(2) There exist vectors $v_{j, \pm}, a_{j, \pm} \in \mathbb{R}^{n}$ such that for $\pm t \geqq t_{0}$,

$$
q_{j}(t)=q_{j, \pm}(t) \equiv t v_{j, \pm}+a_{j, \pm} j=1,2, \ldots, N .
$$

(3) $v_{j, \pm} \neq v_{k, \pm}$ if $j \neq k$.

Assumption II. For any $j=1,2, \ldots, N, V_{j}(x)$ is a real valued function on $\mathbb{R}^{n}$ such that there exist functions $W_{j, 1} \in W^{1, s}\left(\mathbb{R}^{n}\right)$ for some $n / 2<s<n, W_{j, 2} \in W^{1, \infty}\left(\mathbb{R}^{n}\right)$ and a constant $1<\delta<3 / 2$ such that

$$
V_{j}(x)=\left(1+|x|^{2}\right)^{-\delta}\left(W_{j, 1}(x)+W_{j, 2}(x)\right) .
$$

Here $W^{k, q}\left(\mathbb{R}^{n}\right)(k \geqq 0$ is an integer, $1 \leqq q \leqq \infty)$ is the Sobolev space (see Yosida [18], p. 55 for the definition).

Remark 1.1. By Sobolev's embedding theorem (Stein [15], p. 124), $W_{j, 1} \in L^{p}\left(\mathbb{R}^{n}\right)$, $1 / p=1 / s-1 / n$. Note $p>n$. Hence by Hölder's inequality $V_{j} \in L^{q}\left(\mathbb{R}^{n}\right)$ for any $n / 2 \delta<q$ $\leqq p ; A_{j}(x)=\left(1+|x|^{2}\right)^{\delta / 2} V_{j}(x) \in L^{q}\left(\mathbb{R}^{n}\right)$ for any $n / \delta<q \leqq p$. In what follows $p$ is always defined by $1 / p=1 / s-1 / n$.

Before stating the last assumption, we state here several preliminary results of Assumption (I) and (II) which can be readily obtained by using the well-known theorems.

(A) Let $H_{0}$ be the unique selfadjoint extention of $-\Delta /\left.2\right|_{C_{0}^{\infty}\left(\mathbb{R}^{n}\right)}$ on the Hilbert space $\mathfrak{H}=L^{2}\left(\mathbb{R}^{n}\right)$. By Remark 1.1, the multiplication operator $V_{j}(x)$ is $H_{0}$-compact (Reed-Simon [13], p. 369). Hence for any $t \in \mathbb{R}^{1}$ and $j=1,2, \ldots, N, H(t)=$ $H_{0}+\sum V_{j}\left(x-q_{j}(t)\right), \quad H_{ \pm}(t)=H_{0}+\sum V_{j}\left(x-q_{j, \pm}(t)\right), \quad H_{j}(t)=H_{0}+V_{j}\left(x-q_{j}(t)\right)$, $H_{j, \pm}(t)=H_{0}+V_{j}\left(x-q_{j, \pm}(t)\right)$ and $H_{j}=H_{0}+V_{j}(x)$ are selfadjoint on $\mathfrak{S}$ with the common domain $W^{2,2}\left(\mathbb{R}^{n}\right)=H^{2}\left(\mathbb{R}^{n}\right)$.

(B) Since $\partial V_{j} / \partial x_{k} \in L^{q}\left(\mathbb{R}^{n}\right)$ for any $n / 2 \delta<q \leqq S$,

$$
\left\|\frac{d}{d t}\left[\left(H_{0}+1\right)^{-1 / 2} V_{j}\left(x-q_{j}(t)\right)\left(H_{0}+1\right)^{-1 / 2}\right]\right\| \leqq C(t)<\infty
$$

(Reed-Simon [11], Theorem X.19 and Theorem X.20). Therefore by Simon [14], Theorem II.27, $-i H(t)$ generates a unique propagator $U(t, s)(-\infty<t, s<\infty)$ :

i) $U(t, s)$ is a unitary operator on $\mathfrak{H}$ and is strongly continuous in $(t, s)$;

ii) $U(t, s) U(s, r)=U(t, r),-\infty<r, s, t<\infty$;

iii) for $f \in D\left(H_{0}^{1 / 2}\right), U(t, s) f \in D\left(H_{0}^{1 / 2}\right)$ and

$$
(\partial / \partial t) U(t, s) f=-i H(t) U(t, s) f,
$$

where the derivative in the L.H.S. of (1.5) is understood as the strong derivative in the space $H^{-1}\left(\mathbb{R}^{n}\right)=$ the dual space of $W^{1,2}\left(\mathbb{R}^{n}\right)$.

The same statements are true for $H_{ \pm}(t)$ and $H_{j, \pm}(t)$ and we write the 
corresponding unitary propagators as $U_{ \pm}(t, s)$ and $U_{j, \pm}(t, s)$, respectively $(j=1,2, \ldots, N)$.

(3) Let us set as $A_{j}(x)=\left(1+|x|^{2}\right)^{\delta / 2} V_{j}(x) B_{j}(x)=\left(1+|x|^{2}\right)^{-\delta / 2}$ and write as $A_{j}$ and $B_{j}$ the corresponding multiplication operators. $\mathbb{R}_{+}^{1}=[0, \infty)$. For Banach space $\mathfrak{X}$, $\mathbf{B}(\mathfrak{X})$ is the Banach algebra of all bounded operators on $\mathfrak{X}$. For a closable operator $T$, $[T]$ is its closure. We write as $r_{0}(z)=\left(H_{0}-z\right)^{-1}, z \notin \mathbb{R}_{+}^{1}$.

(i) (Ginibre-Moulin [4], Prop. 3.1.) Let $Q_{j}(z)=\left[A_{j}\left(H_{0}-z\right)^{-1} B_{j}\right]$ for $z \in \mathbb{C}^{1} \backslash \mathbb{R}_{+}^{1}$. Then $Q_{j}(z)$ is a $\mathbf{B}(\mathfrak{H})$-valued analytic function there and can be extended to the closed cut plane (the closure of $\mathbb{C}^{1} \backslash \mathbb{R}_{+}^{1}$ where upper and lower boundaries are distinguished) as a $\mathbf{B}(\mathfrak{H})$-valued Hölder continuous function. $\left\|Q_{j}(z)\right\| \rightarrow 0$ as $|z| \rightarrow \infty$. We write its boundary values on $\mathbb{R}_{+}^{1}$ as $Q_{j}(\lambda \pm i 0)=\lim _{\varepsilon \downarrow 0} Q_{j}(\lambda \pm i \varepsilon)$.

(ii) (Konno-Kuroda [9].) For any $j=1,2, \ldots, N, H_{j}$ has at most finite number of negative eigenvalues of finite multiplicity. We write the eigenvalues and the corresponding eigenfunctions of $H_{j}$ as $\mu_{j, 1}, \ldots, \mu_{j, m_{j}}$ and $\phi_{j, 1}, \ldots, \phi_{j, m_{j}}\left(\phi_{j, k}\right.$ is normalized). Clearly $H_{j, \pm}(t)$ has the same eigenvalues and the eigenfunctions $\phi_{j, \pm, t, k}=\phi_{j, k}\left(x-q_{j, \pm}(t)\right), k=1, \ldots, m_{j}$. We write the projection onto the closed subspace spanned by $\phi_{j, \pm, t, k}$ 's as $P_{j, \pm}(t)$.

(iii) (Agmon [1], Lemma 4.2.) For any multi-index $|\alpha| \leqq 2$ and any $\rho \geqq 0$,

$$
\begin{aligned}
& \left(1+|x|^{2}\right)^{\rho}(\partial / \partial x)^{\alpha} \phi_{j, k} \in L^{2}\left(\mathbb{R}^{n}\right), \\
& j=1,2, \ldots, N, \quad k=1,2, \ldots, m_{j} .
\end{aligned}
$$

Assumption III. For any $j=1,2, \ldots, N, I+Q_{j}(\lambda \pm i 0)$ has its inverse in $\mathbf{B}(\mathfrak{G})$ for $\lambda \geqq 0$.

Remark. By this assumption we assume that $H_{j}\left(\right.$ or $\left.H_{j, \pm}(t)\right)$ has no non-negative eigenvalues or resonances.

Now we can state our main theorem in this paper.

Theorem. Let Assumptions (I), (II) and (III) be satisfied. Then for any $s \in \mathbb{R}^{1}$, the following statements hold.

(1) (Existence of the wave operators.) The following limits exist:

$$
\begin{aligned}
& s-\lim _{t \rightarrow \pm \infty} U(t, s)^{-1} \exp \left(-i(t-s) H_{0}\right)=W_{0, \pm}(s) \\
& s-\lim _{t \rightarrow \pm \infty} U(t, s)^{-1} U_{j, \pm}(t, s) \exp \left(i x \cdot v_{j, \pm}\right) P_{j, \pm}(s)=W_{j, \pm}(s) .
\end{aligned}
$$

(2) (Asymptotic orthogonality.) The ranges $R\left(W_{j, \pm}(s)\right), j=0,1, \ldots, N$, are orthogonal each other.

(3) (Asymptotic completeness.)

$$
\bigoplus_{j=0}^{N} R\left(W_{j, \pm}(s)\right)=\mathfrak{H} .
$$

The rest of this paper is devoted to the proof of this theorem. We sketch here the outline of the proof with somewhat crude terminology, displaying the plan of this paper. In Sect. 2, we shall prove the existence and the asymptotic orthogonality of 
the wave operators by standard methods (Reed-Simon [12], Sect. XI.3, XI.5). We shall prove the asymptotic completeness in Sect. 3 which is devided into five subsections. To prove the completeness we must make a detour. According to Howland [5], we shall introduce bigger Hilbert spaces $\Re_{1}=L^{2}\left(\mathbb{R}_{t}^{1}\right.$, $\left.L^{2}\left(\mathbb{R}_{x}^{n}\right)\right) \oplus L^{2}\left(\mathbb{R}_{t}^{1}\right) \oplus \cdots \oplus L^{2}\left(\mathbb{R}_{t}^{1}\right)$ and $\Omega_{2}=L^{2}\left(\mathbb{R}_{t}^{1}, L^{2}\left(\mathbb{R}_{x}^{n}\right)\right)$ of square integrable functions of time-space variables $(t, x): \Omega_{1}$ is the "channel Hilbert space" and $\boldsymbol{\Omega}_{2}$ is the "basic space". The channel Hamiltonian $K_{1}=\left(-i \partial / \partial t+H_{0}\right) \oplus \sum_{j, k} \oplus(-i \partial / \partial t$ $\left.+\mu_{j, k}\right)$ is considered on $\Omega_{1}$ and the Hamiltonain $K_{2}=-i \partial / \partial t+H(t)$ is considered on $\Omega_{2}$. Then we shall study the two space scattering between $K_{1}$ and $K_{2}$ via the identification operator $J: \mathfrak{R}_{1} \rightarrow \mathfrak{R}_{2}, J$ is defined by (3.2). It will be proved in

Subsect. 3.1 that the wave operators $\mathscr{W}_{ \pm}=s-\lim \exp \left(i \tau K_{2}\right) J \exp \left(-i \tau K_{1}\right)$ exist and are the isometries; the completeness of $\mathscr{W}_{ \pm}$implies that of the original wave operators. Thus by eliminating the explicit time dependence of the Hamiltonians by this procedure, the problem is reduced to the completeness problem of the wave operator $\mathscr{W}_{ \pm}$for the time-independent Hamiltonians. Here is an important observation: If we replace - $i \partial / \partial t$ by the kinetic energy $-\Delta_{y} / 2$ of certain particle and $q_{j}(t)$ 's by y in $K_{1}$ and $K_{2}$, then $K_{1}$ and $K_{2}$ have the same form as the Hamiltonians appearing in three body scattering theory (see Faddeev [3], Ginibre-Moulin [4], Howland [6], Kato [8] and Yajima [17]). Being suggested by this observation, we shall prove the completeness of $\mathscr{W}_{ \pm}$by using the methods of three body problem. In Subsect. 3.2, we record the abstract theorem due to Kato [8] by which the completeness will be finally proved. In Subsect. 3.3, an algebraic procedure of the construction of the substitute of the "Faddeev matrix" will be carried out in a way similar to that of Howland [5] in three body case. Various estimates of the operators necessary to apply Kato's theorem will be done in Subsect. 3.4. The proof of the completeness of the original wave operators will be completed in Subsect. 3.5.

The following notation and conventions are used throughout the paper. For $1 \leqq q \leqq \infty, L^{q}\left(\mathbb{R}^{n}\right)$ is the Banach space of all $q$-summable functions on $\mathbb{R}^{n}$ with natural norm. For non-negative integer $k, W^{k, q}\left(\mathbb{R}^{n}\right)$ is the Sobolev space, $H^{k}\left(\mathbb{R}^{n}\right)=W^{k, 2}\left(\mathbb{R}^{n}\right)$. For $\gamma \in \mathbb{R}^{1}, L_{\gamma}^{2}\left(\mathbb{R}^{n}\right)$ is the weighted $L^{2}$-space:

$$
L_{\gamma}^{2}\left(\mathbb{R}^{n}\right)=\left\{f \in L_{\mathrm{loc}}^{2}\left(\mathbb{R}^{n}\right):\left\|\left(1+x^{2}\right)^{\gamma / 2} f\right\|_{L^{2}}=\|f\|_{L_{r}^{2}}<\infty\right\} .
$$

The norm of $L^{2}\left(\mathbb{R}^{n}\right)$ is usually written as $\|\quad\|$ regardless of the dimension of the space $\mathbb{R}^{n}$; the norms of other spaces are denoted as \|\|$_{L^{q}},\|\|_{W^{k, q}}$ and etc.

For multi-index $\alpha=\left(\alpha_{1}, \alpha_{2}, \ldots, \alpha_{n}\right), \alpha_{j} \in \mathbb{N}, \quad(\partial / \partial x)^{\alpha}=\left(\partial / \partial x_{1}\right)^{\alpha_{1}} \ldots\left(\partial / \partial x_{n}\right)^{\alpha_{n}}$; $x^{\alpha}=x_{1}^{\alpha_{1}} \ldots x_{n}^{\alpha_{n}}$. For multi-induces $\alpha$ and $\beta, \alpha_{\leqq} \beta$ means $\alpha_{j} \leqq \beta_{j}$ for all $j=1, \ldots, n$; if $\alpha \leqq \beta,\left(\begin{array}{l}\alpha \\ \beta\end{array}\right)=\left(\begin{array}{l}\alpha_{1} \\ \beta_{1}\end{array}\right) \ldots\left(\begin{array}{l}\alpha_{n} \\ \beta_{n}\end{array}\right)$, where for $a, b \in \mathbb{N},\left(\begin{array}{l}a \\ b\end{array}\right)=\frac{a !}{b !(a-b) !} \cdot \mathbb{C}_{ \pm}=\{z \in \mathbb{C}$ : $\operatorname{Im} z \gtrless 0\}$ and for $I \subset \mathbb{R}^{1}, \mathbb{C}_{+}(I)=\left\{z \in \mathbb{C}_{+}: \operatorname{Re} z \in I\right\} . \mathscr{F}_{x}$ (or $\mathscr{F}_{t}$ ) is the Fourier transform with respect to the variable $x$ (or $t$ ). We write $\mathscr{F} f=\hat{f}$, regardless of the variable.

For Hilbert spaces $\mathfrak{H}_{1}, \ldots, \mathfrak{H}_{m}, \mathfrak{H}_{1} \oplus \cdots \oplus \mathfrak{H}_{m}$ and $\mathfrak{H}_{1} \otimes \cdots \otimes \mathfrak{S}_{m}$ are their direct product and tensor product. If there exists a linear topological space $\mathfrak{Q}$ such that $\mathfrak{H}_{j} \subset \mathfrak{Q}$ for any $j=1, \ldots, m, \mathfrak{H}_{1}+\cdots+\mathfrak{H}_{m}$ is the sum space of $\mathfrak{H}_{j}$ 's. If $A_{1}, \ldots, A_{m}$ 
are closed operators on $\mathfrak{H}_{1}, \ldots, \mathfrak{H}_{m}$, respectively, $A_{1} \oplus \cdots \oplus A_{m}$ and $A_{1} \otimes \cdots \otimes A_{m}$ are their direct product and tensor product. $A_{1} \otimes I$ and $I \otimes A_{2}$ are often written as $A_{1}$ and $A_{2}$ simply. Identity operator is often written as 1 . If $A$ is a one-one closed operator from $\mathfrak{S}_{1}$ to $\mathfrak{H}_{2}$, the range $R(A)$ of $A$ is considered as a Hilbert space with the norm $\|A u\|_{R(A)}=\left(\|A u\|^{2}+\|u\|^{2}\right)^{1 / 2}$. For a family of Hilbert spaces $\{\mathfrak{G}(t)$ :

$-\infty<t<\infty\}, \int^{\oplus} \mathfrak{H}(t) d t$ is the direct integral of $\{\mathfrak{H}(t)\}$. For a closable operator $A$, $[A]$ is its closure. If $[A]$ is a bounded operator we often use the notation as if the operator $A$ itself is a bounded one. For Banach spaces $\mathfrak{X}$ and $\mathfrak{Y}, \mathbf{B}(\mathfrak{X}, \mathfrak{Y})$ is the space of all bounded operators from $\mathfrak{X}$ to $\mathfrak{Y}, \mathbf{B}_{\infty}(\mathfrak{X}, \mathfrak{Y})$ the compact operators from $\mathfrak{X}$ to $\mathfrak{Y}$, $\mathbf{B}(\mathfrak{X})=\mathbf{B}(\mathfrak{X}, \mathfrak{X})$. The symbol $\oplus$ is also used to denote the sum of orthogonal elements in a Hilbert space.

If $m(x)$ is a function, the same symbol $m(x)$ is also used to denote the multiplication operator by the function. The integral without referring to the region of integration is understood to be taken over the whole region of the variable.

\section{Existence and Asymptotic Completeness}

Here we shall prove the first two statements of the theorem. We start with the following lemma.

Lemma 2.1. Let Assumptions $I$ and II be satisfied. Then for any $s \in \mathbb{R}^{1}$ the following statements hold.

(1) The following limits exist:

$$
\begin{aligned}
& \underset{s-\lim }{s \rightarrow \pm \infty} U(t, s)^{-1} U_{ \pm}(t, s)=\Gamma_{ \pm}(s) \\
& s-\lim _{t \rightarrow \pm \infty} U_{ \pm}(t, s)^{-1} U(t, s)=\Omega_{ \pm}(s) .
\end{aligned}
$$

(2) $\Gamma_{ \pm}(s)$ and $\Omega_{ \pm}(s)$ are unitary operators on $\mathfrak{S}$ and $\Gamma_{ \pm}(s)=\Omega_{ \pm}(s)^{-1}$.

Proof. Since $H(t)=H_{ \pm}(t)$ for $\pm t \geqq t_{0}, U\left(t_{2}, t_{1}\right)=U_{ \pm}\left(t_{2}, t_{1}\right)$ if $\pm t_{1} \geqq t_{0}$ and $\pm t_{2} \geqq t_{0}$. Therefore if $\pm t \geqq t_{0}$,

$$
\begin{aligned}
& U(t, s)^{-1} U_{ \pm}(t, s)=U\left(t_{0}, s\right)^{-1} U_{ \pm}\left(t_{0}, s\right) ; \\
& U_{ \pm}(t, s)^{-1} U(t, s)=U_{ \pm}\left(t_{0}, s\right)^{-1} U\left(t_{0}, s\right),
\end{aligned}
$$

by Sect. 1, (B), ii). Thus (1) holds trivially. (2) is an immediate consequence of (1).

By Lemma 2.1 and the chain rule for the wave operators (Reed-Simon [12], p. 18 ), it suffices to prove the theorem under the condition that $q_{j}(t)=q_{j,+}(t)$ for "+" case $(t \rightarrow \infty)$, and $q_{j}(t)=q_{j,-}(t)$ for " - " case $(t \rightarrow-\infty)$. Since the following argument for " +" case equally applies to " - " case, we shall treat the " + " case only. Thus we assume hereafter that $H(t)=H_{+}(t), H_{j}(t)=H_{j,+}(t)$ and we write $v_{j,+}$, etc. as $v_{j}$, etc., omitting the suffix " $+"$. Since the cases $a_{j} \neq 0$ can be treated similarly, we assume $a_{j}=0(j=1,2, \ldots, N)$ hereafter. 
Lemma 2.2. Let $\mathrm{F}, \mathrm{G}$ be the multiplication operators by $f, g \in L^{q}\left(\mathbb{R}^{n}\right)(1 \leqq q \leqq \infty)$ and $u \in \mathfrak{H}$. Then

(1) $\left\|F e^{-i t H_{0}} G u\right\| \leqq(2 \pi|t|)^{-n / q}\|f\|_{L^{q}}\|g\|_{L^{q}}\|u\|$;

(2) $\lim _{t \rightarrow \pm \infty}\left\|\left(e^{-i t H_{0}} u\right)(x)-|t|^{-n / 2} e^{i\left(x^{2} / 2 t-n \pi / 4\right)} \hat{u}(x / t)\right\|=0$.

Statement (1) is proved by Kato [7], p. 277 and statement (2) is Theorem IX. 31 of Reed-Simon [11].

Lemma 2.3. For any $t, s \in \mathbb{R}^{1}, j=1,2, \ldots, N$ and $k=1,2, \ldots, m_{j}$,

$$
\begin{aligned}
& \left(U_{j}(t, s)\left(\exp \left(i x \cdot v_{j}\right) \phi_{j, s, k}\right)\right)(x) \\
& \quad=\exp \left(i x \cdot v_{j}-i(t-s)\left(v_{j}^{2} / 2+\mu_{j, k}\right)\right) \phi_{j, s, k}\left(x-(t-s) v_{j}\right) .
\end{aligned}
$$

Proof. If $t=s,(2.1)$ obviously holds. By using the equation $H_{j}(t) \phi_{j, s, k}\left(x-(t-s) v_{j}\right)$ $=\mu_{j, k} \phi_{j, s, k}\left(x-(t-s) v_{j}\right)$, we get by direct calculations that for any $s \in \mathbb{R}^{1}$

$$
\left(-i \partial / \partial t+H_{j}(t)\right)\left(\exp \left(i x \cdot v_{j}-i(t-s)\left(v_{j}^{2} / 2+\mu_{j, k}\right)\right) \phi_{j, s, k}\left(x-(t-s) v_{j}\right)\right)=0 .
$$

Then the uniqueness of the propagator (Sect. 1, (B)) shows that (2.1) holds.

(Q.E.D.)

Proof of Statement (1). We prove the case $s=0$ only. The other cases can be proved similarly. We use the Cook's method (Reed-Simon [12], Theorem XI.4).

(i) We first prove the existence of the limit (1.7). Let $f, g \in C_{0}^{\infty}\left(\mathbb{R}^{n}\right)$. Then $\left(U(t, 0)^{-1} \exp \left(-i t H_{0}\right) f, g\right)=\left(\exp \left(-i t H_{0}\right) f, U(t, 0) g\right)$ is continuously differentiable with respect to $t$, since $\exp \left(-i t H_{0}\right) f \in D\left(H_{0}\right)$ and $U(t, 0) g$ is continuously differentiable in $H^{-1}\left(\mathbb{R}^{n}\right)$ by Sect. 1, (B), iii). Hence

$$
\begin{aligned}
& \left(U\left(t_{2}, 0\right)^{-1} \exp \left(i t_{2} H_{0}\right) f, g\right)-\left(U\left(t_{1}, 0\right)^{-1} \exp \left(-i t_{1} H_{0}\right) f, g\right) \\
& \quad=\int_{t_{1}}^{t_{2}} \frac{d}{d \sigma}\left(\exp \left(-i \sigma H_{0}\right) f, U(\sigma, 0) g\right) d \sigma \\
& \quad=\sum_{j=1}^{N} i \int_{t_{1}}^{t_{2}}\left(U(\sigma, 0)^{-1} V_{j}\left(x-\sigma v_{j}\right) \exp \left(-i \sigma H_{0}\right) f, g\right) d \sigma .
\end{aligned}
$$

By Schwartz's inequality we have

$$
\begin{aligned}
& \left|\left(U\left(t_{2}, 0\right)^{-1} \exp \left(-i t_{2} H_{0}\right) f-U\left(t_{1}, 0\right)^{-1} \exp \left(-i t_{1} H_{0}\right) f, g\right)\right| \\
& \leqq \sum_{j=1}^{N} \int_{t_{1}}^{t_{2}}\left\|V_{j}\left(x-\sigma v_{j}\right) \exp \left(-i \sigma H_{0}\right) f\right\| d \sigma\|g\|,
\end{aligned}
$$

which obviously implies

$$
\begin{gathered}
\left\|U\left(t_{2}, 0\right)^{-1} \exp \left(-i t_{2} H_{0}\right) f-U\left(t_{1}, 0\right)^{-1} \exp \left(-i t_{1} H_{0}\right) f\right\| \\
\leqq \sum_{j=1}^{N} \int_{t_{1}}^{t_{2}}\left\|V_{j}\left(x-\sigma v_{j}\right) \exp \left(-i \sigma H_{0}\right) f\right\| d \sigma .
\end{gathered}
$$

Now take $q$ as $n / 2 \delta \leqq q<n / 2$. Then by Hölder's inequality $\| V_{j}\left(x-\sigma v_{j}\right)$ 
$\exp \left(-i \sigma H_{0}\right) f\|\leqq\| V_{j}\left\|_{q}\right\| \exp \left(-i \sigma H_{0}\right) f \|_{r}, 1 / r+1 / q=1 / 2$. Applying Kato's estimate (Kato [7]), we have $\left\|\exp \left(-i \sigma H_{0}\right) f\right\|_{r} \leqq(2 \pi|\sigma|)^{-n / q}\|f\|_{r^{\prime}}, 1 / r+1 / r^{\prime}=1$. Thus we have $\left\|V_{j}\left(x-\sigma v_{j}\right) \exp \left(-i \sigma H_{0}\right) f\right\| \leqq\left\|V_{j}\right\|_{q}\|f\|_{r^{\prime}}(2 \pi|\sigma|)^{-n / q}$. Since $n / 2 \delta \leqq$ $q<n / 2$, the integrand in the R.H.S. of $(2.2)$ is integrable on $(1, \infty)$. Therefore $U(t, 0)^{-1} \exp \left(-i t H_{0}\right) f$ is convergent as $t \rightarrow \infty$. Since $U(t, 0)^{-1} \exp \left(-i t H_{0}\right)$ is unitary for any $0 \leqq t<\infty$, this holds for any $f \in \mathfrak{H}$.

(ii) Next we prove the existence of (1.8). Obviously it suffices to prove that $U(t, 0)^{-1} U_{j}(t, 0)\left(\exp \left(i x \cdot v_{j}\right) \phi_{j, 0, k}\right)$ is convergent as $t \rightarrow \infty$. We omit the suffix 0 in $\phi_{j, 0, k}$ hereafter. By a similar argument as to derive (2.2), we have

$$
\begin{gathered}
\| U\left(t_{2}, 0\right)^{-1} U_{j}\left(t_{2}, 0\right)\left(\exp \left(i x \cdot v_{j}\right) \phi_{j, k}\right) \\
-U\left(t_{1}, 0\right)^{-1} U_{j}\left(t_{1}, 0\right)\left(\exp \left(i x \cdot v_{j}\right) \phi_{j, k}\right) \| \\
\leqq \sum_{l \neq j} \int_{t_{1}}^{t_{2}}\left\|V_{l}\left(x-\sigma v_{l}\right) U_{j}(\sigma, 0)\left(\exp \left(i x \cdot v_{j}\right) \phi_{j, k}\right)\right\| d \sigma .
\end{gathered}
$$

By (2.1), $\quad\left\|V_{l}\left(x-\sigma v_{l}\right) U_{j}(\sigma, 0)\left(\exp \left(i x \cdot v_{j}\right) \phi_{j, k}\right)\right\|=\left\|V_{l}\left(x-\sigma v_{l}\right) \phi_{j, k}\left(x-\sigma v_{j}\right)\right\|=$ $\left\|V_{l}(x) \phi_{j, k}\left(x-\sigma\left(v_{j}-v_{l}\right)\right)\right\|$. We write $v_{j}-v_{l}=v \neq 0$. Then by Hölder's inequality $\left\|V_{l}(x) \phi_{j, k}(x-\sigma v)\right\| \leqq\left\|V_{l}(x)\left(1+|x|^{2}\right)^{\delta / 2}\right\|_{L_{n}} \|\left(1+|x|^{2}\right)^{-\delta / 2} \times$ $\left(1+|x-\sigma v|^{2}\right)^{-\delta / 2}\left\|_{L^{\infty}}\right\|\left(1+|x-\sigma v|^{2}\right)^{\delta / 2} \phi_{j, k}(x-\sigma v) \|_{L^{r}}, \quad 1 / r+1 / n=1 / 2 . \quad$ By Remark $1.1\left\|V_{l}(x)\left(1+|x|^{2}\right)^{\delta / 2}\right\|_{L^{n}}<\infty$; by (1.6) and Sobolev's embedding theorem $\left\|\left(1+|x|^{2}\right)^{\delta / 2} \phi_{j, k}(x)\right\|_{L^{r}}<\infty$. On the other hand we have by elementary computation that $\left\|\left(1+|x|^{2}\right)^{-\delta / 2}\left(1+|x-\sigma v|^{2}\right)^{-\delta / 2}\right\|_{L^{\infty}} \leqq 2^{-\delta / 2}\left(1+|\sigma v|^{2}\right)^{-\delta / 2}$. Since $\delta>1$, the R.H.S. of $(2.3)$ is integrable on $(1, \infty)$. Hence $U(t, 0)^{-1} U_{j}(t, 0)\left(\exp \left(i x \cdot v_{j}\right) \phi_{j, k}\right)$ is convergent as $t \rightarrow \infty$. (Q.E.D.)

Proof of Asymptotic Orthogonality. Again we prove the case $s=0$ only. Other cases can be proved similarly. For $j, k=0,1,2, \ldots, N$,

$$
\left(W_{j}(0) f, W_{k}(0) g\right)=\lim _{t \rightarrow \infty}\left(U_{j}(t, 0) e^{i x \cdot v_{j}} P_{j}(0) f, U_{k}(t, 0) e^{i x \cdot v_{k}} P_{k}(0) g\right),
$$

where $v_{0}=0, P_{0}(0)=I$ and $U_{0}(t, s)=\exp \left(-i(t-s) H_{0}\right)$. Therefore it suffices to prove

$$
\begin{aligned}
& \lim _{t \rightarrow \infty}\left(U_{0}(t, 0) f, U_{j}(t, 0)\left(\exp \left(i x \cdot v_{j}\right) \phi_{j, k}\right)\right)=0 \\
& \lim _{t \rightarrow \infty}\left(U_{j}(t, 0)\left(\exp \left(i x \cdot v_{j}\right) \phi_{j, k}\right), U_{l}(t, 0)\left(\exp \left(i x \cdot v_{l}\right) \phi_{l, m}\right)\right)=0,
\end{aligned}
$$

for $j, l=1,2, \ldots, N, k=1,2, \ldots, m_{j}, m=1,2, \ldots, m_{m}, j \neq l$ and $f \in C_{0}^{\infty}\left(\mathbb{R}^{n}\right)$. Since

$$
\begin{aligned}
& \left|\left(U_{j}(t, 0)\left(\exp \left(i x \cdot v_{j}\right) \phi_{j, k}\right), U_{l}(t, 0)\left(\exp \left(i x \cdot v_{l}\right) \phi_{l, m}\right)\right)\right| \\
& \quad \leqq \int_{\mathbb{R}^{n}}\left|\phi_{j, k}\left(x-v_{j} t\right) \phi_{l, m}\left(x-v_{l} t\right)\right| d x,
\end{aligned}
$$

(2.5) obviously holds. By Lemma 2.2, (2) and (1.6),

$$
\begin{aligned}
& \limsup _{t \rightarrow \infty}\left|\left(\exp \left(-i t H_{0}\right) f, U_{j}(t, 0)\left(\exp \left(i x \cdot v_{j}\right) \phi_{j, k}\right)\right)\right| \\
& \quad \leqq \limsup _{t \rightarrow \infty} \int_{\mathbb{B}^{n}} t^{-n / 2}\left|\hat{f}(x / t) \phi_{j, k}\left(x-v_{j} t\right)\right| d x
\end{aligned}
$$




$$
\begin{aligned}
& \leqq \limsup _{t \rightarrow \infty} t^{-n / 2}\|\hat{f}\|_{L^{\infty}}\left\|\left(1+|x|^{2}\right)^{(n+1) / 2} \phi_{j, k}\right\|\left\|\left(1+|x|^{2}\right)^{-(n+1) / 2}\right\| \\
& =0 .
\end{aligned}
$$

This proves (2.4)

(Q.E.D.)

\section{Asymptotic Completeness}

This section is devoted to the proof of the asymptotic completeness of the wave operators, the third statement of the theorem.

\subsection{Reduction to the Stationary Problem}

According to Howland [5], we shall prove the asymptotic completeness, reducing the problem to the stationary one. Let us first introduce two accessory Hilbert spaces $\boldsymbol{R}_{1}$ and $\boldsymbol{R}_{2}$ as follows.

$$
\left\{\begin{array}{l}
\mathfrak{R}_{1}=\left(L^{2}\left(\mathbb{R}^{1}\right) \otimes L^{2}\left(\mathbb{R}^{n}\right)\right) \oplus L^{2}\left(\mathbb{R}^{1}\right) \oplus L^{2}\left(\mathbb{R}^{1}\right) \oplus \ldots \oplus L^{2}\left(\mathbb{R}^{1}\right), \sum m_{j} \text {-copies } \\
\mathfrak{\Re}_{2}=L^{2}\left(\mathbb{R}^{1}\right) \otimes L^{2}\left(\mathbb{R}^{n}\right),
\end{array}\right.
$$

We write the generic element of $\boldsymbol{\Omega}_{1}$ as $\tilde{u}={ }^{\dagger}\left(u, \sigma_{1,1}, \ldots, \sigma_{N, m_{N}}\right)$, where ${ }^{\dagger}$ stands for the transpose. We define the identification operator $J$ from $\Re_{1}$ to $\Omega_{2}$ as

$$
J \tilde{u}=u+\sum_{j=1}^{N} T_{v_{j}}\left(\sum_{k=1}^{m_{j}} \sigma_{j, k} \otimes \phi_{j, k}\right),
$$

where for any vector $v \in \mathbb{R}^{n}$ the operator $T_{v}$ is defined as

$$
\left(T_{v} f\right)(t, x)=\exp \left(i\left(x \cdot v-v^{2} t / 2\right)\right) f(t, x-v t)
$$

for $f \in L^{2}\left(\mathbb{R}^{1}\right) \otimes L^{2}\left(\mathbb{R}^{n}\right)=L^{2}\left(\mathbb{R}^{n+1}\right)$. Obviously $J \in \mathbf{B}\left(\Re_{1}, \Re_{2}\right)$. Now we define one parameter families of operators $\mathscr{U}_{1}(\tau)$ and $\mathscr{U}_{2}(\tau)(-\infty<\tau<\infty)$ on the spaces $\Re_{1}$ and $\Omega_{2}$, respectively as follows:

$$
\begin{aligned}
\left(\mathscr{U}_{1}(\tau) \tilde{u}\right)(t)= & \left(\exp \left(-i \tau H_{0}\right) u(t-\tau), \exp \left(-i \tau \mu_{1,1}\right) \sigma_{1,1}(t-\tau)\right. \\
& \left.\ldots, \exp \left(-i \tau \mu_{N, m_{N}}\right) \sigma_{N, m_{N}}(t-\tau)\right) ; \\
\left(\mathscr{U}_{2}(\tau) u\right)(t)= & U(t, t-\tau) u(t-\tau)
\end{aligned}
$$

$\mathscr{U}_{1}(\tau)$ is obviously a strongly continuous unitary group on $\Re_{1}$ and so is $\mathscr{U}_{2}(\tau)$ on $\boldsymbol{S}_{2}$ since $U(t, s)$ is strongly continuous in both variables $t$ and $s$ and is unitary. Hence by Stone's theorem there exist selfadjoint operators $K_{1}$ on $\Re_{1}$ and $K_{2}$ on $\Re_{2}$ such that

$$
\mathscr{U}_{1}(\tau)=\exp \left(-i \tau K_{1}\right), \quad \mathscr{U}_{2}(\tau)=\exp \left(-i \tau K_{2}\right) .
$$

By (3.3) we readily see that

$$
\begin{aligned}
& K_{1}=L_{0} \oplus \sum_{j=1}^{N} \oplus\left(\sum_{k=1}^{m_{j}} \oplus L_{j, k}\right), \\
& L_{0}=-i \partial / \partial t+H_{0} ; \quad L_{j, k}=-i \partial / \partial t+\mu_{j, k} .
\end{aligned}
$$

The following lemma plays an import role. 
Lemma 3.1. Let Assumption (I) and (II) be satisfied. Then the following statements hold.

(1) For any $\tilde{u} \in \mathfrak{R}_{1}$,

$$
\lim _{\tau \rightarrow \infty}\left\|J \exp \left(-i \tau K_{1}\right) \tilde{u}\right\|=\|\tilde{u}\| .
$$

(2) The following limit exists:

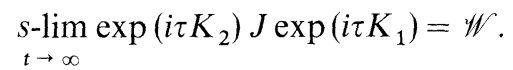

(3) The operator $\mathscr{W}$ is isometry from $\mathfrak{R}_{1}$ to $\mathfrak{\Omega}_{2}$.

(4) If the range $R(\mathscr{W})=\mathfrak{S}_{2}$, the statement (3) of the theorem holds.

Proof. By definition

$$
\begin{aligned}
& J \exp \left(-i \tau K_{1}\right) \tilde{u}(t) \\
& \quad=e^{-i \tau H_{0}} u(t-\tau)+\sum_{j=1}^{N} T_{v_{j}}\left(\sum_{k=1}^{m_{j}} e^{-i \tau \mu_{j},{ }^{k}} \sigma_{j, k}(\cdot-\tau) \otimes \phi_{j, k}\right)(t) .
\end{aligned}
$$

Each term under the $\sum$-sign in the R.H.S. of (3.7) can be written by (2.1) as

$$
\begin{aligned}
& e^{i\left(x \cdot v_{j}-t v_{j}^{2} / 2\right)} \phi_{j, k}\left(x-v_{j} t\right) e^{-i \tau \mu_{j}, k} \sigma_{j, k}(t-\tau) \\
& \quad=e^{-i(\tau-t) \mu_{j}, k} \sigma_{j, k}(t-\tau) \otimes\left(U_{j}(t, 0) e^{i x \cdot v_{j}} \phi_{j, k}\right) .
\end{aligned}
$$

We prove (1) first. Since $T_{v}$ is unitary, it suffices to prove that for $j, l=1,2, \ldots, N$, $k=1,2, \ldots, m_{j}, m=1,2, \ldots, m_{l}$ such that $(j, k) \neq(l, m)$,

$$
\begin{aligned}
& \lim _{\tau \rightarrow \infty}\left(e^{-i t H_{0}} u(t-\tau), \phi_{j, k}\left(x-v_{j} t\right) e^{-i\left(\tau \mu_{j, k}-x \cdot v_{j}+t v_{j}^{2} / 2\right)} \sigma_{j, k}(t-\tau)\right)_{\Omega_{2}}=0 ; \\
& \lim _{\tau \rightarrow \infty}\left(\phi_{j, k}\left(x-v_{j} t\right) e^{-i\left(\tau \mu_{j \cdot k}-x \cdot v_{j}+t v_{j}^{2} / 2\right)} \sigma_{j, k}(t-\tau),\right. \\
& \left.\quad \phi_{l, m}\left(x-v_{l} t\right) e^{-i\left(\tau \mu_{l, m}-x \cdot v_{l}+t v_{t}^{2} / 2\right)} \sigma_{l, m}(t-\tau)\right)_{\Omega_{2}}=0 .
\end{aligned}
$$

Let us prove (3.9) first. The inner product is majorized by

$$
\begin{aligned}
& \int d t \int d x\left|\left(e^{-i \tau H_{0}} u(t-\tau, \cdot)\right)(x) \phi_{j, k}\left(x-v_{j} t\right) \sigma_{j, k}(t-\tau)\right| \\
& \quad=\int\left|\sigma_{j, k}(t)\right| d t \int d x\left|\left(e^{-i \tau H_{0}} u(t, \cdot)\right)(x) \phi_{j, k}\left(x-v_{j}(t+\tau)\right)\right| .
\end{aligned}
$$

By Schwartz's inequality the integral by $x$ in the R.H.S. of (3.11) is majorized by $\|u(t,)$.$\| which is square integrable with respect to t$. On the other hand if we fix $t$, this integral coverges to zero as $\tau \rightarrow \infty$. This can be proved exactly in the same way as to prove (2.4). Hence (3.9) is an immediate consequence of Lebesgue's dominated convergence theorem. Next we prove (3.10). If $j=l$ and $k \neq m,(3.10)$ is obvious, since $\phi_{j, k}$ and $\phi_{l, m}$ are orthogonal each other then. Suppose $j \neq l$. The inner product is majorized by

$\int d t\left|\sigma_{j, k}(t) \sigma_{l, m}(t)\right| \int d x\left|\phi_{j, k}\left(x-v_{j}(t+\tau)\right) \phi_{l, m}\left(x-v_{l}(t+\tau)\right)\right|$. 
The integral by $x$ in (3.12) is uniformly bounded by 1 ; for each fixed $t \in \mathbb{R}^{1}$ this converges to zero as $\tau \rightarrow \infty$, since $\phi_{j, k}$ and $\phi_{l, m}$ are normalized and $v_{j} \neq v_{l}$. Thus (3.10) is an immediate consequence of Lebesgue's dominated convergence theorem. Next we prove (2). By (3.4), (3.7) and (3.8) we have

$$
\begin{aligned}
(\exp & \left.\left(i \tau K_{2}\right) J \exp \left(-i \tau K_{1}\right) \tilde{u}\right)(t) \\
= & U(t, t+\tau)\left(J \exp \left(-i \tau K_{1}\right) \tilde{u}\right)(t+\tau) \\
= & U(t, 0)\left[U(0, t+\tau) e^{-i(t+\tau) H_{0}} e^{i t H_{0}} u(t)\right. \\
& \left.\quad+\sum_{j, k} e^{i t \mu_{j, k}} \sigma_{j, k}(t) \otimes U(0, t+\tau) U_{j}(t+\tau, 0) e^{i x \cdot v_{j}} \phi_{j, k}\right]
\end{aligned}
$$

By (3.13) and statement (1) of the theorem, for any fixed $t \in \mathbb{R}^{1}$, we have

$$
\begin{aligned}
& s-\lim _{\tau \rightarrow \infty}\left(\exp \left(i \tau K_{2}\right) J \exp \left(-i \tau K_{1}\right) \tilde{u}\right)(t) \\
& \quad=U(t, 0)\left[W_{0}(0) e^{i t H_{0}} u(t)+\sum_{j, k} e^{i t \mu_{j, k}} \sigma_{j, k}(t) \otimes W_{j}(0) \phi_{j, k}\right],
\end{aligned}
$$

where the limit is understood in the sense of the strong convergence in $\mathfrak{H}$. Here in the R.H.S. of (3.14) all summands are orthogonal each other by statement (2) of the theorem and the isometry property of $W_{j}(0)$ 's. On the other hand $\left\|\left(\exp \left(i \tau K_{2}\right) J \exp \left(-i \tau K_{1}\right) \tilde{u}\right)(t)\right\| \leqq\|u(t)\|+\sum\left|\sigma_{j, k}(t)\right|$, and the R.H.S. of (3.14) is also majorized by $\|u(t)\|+\sum\left|\sigma_{j, k}(t)\right|$. Since $\left(\|u(t)\|+\sum\left|\sigma_{j, k}(t)\right|\right)^{2}$ is integrable with respect to $t \in \mathbb{R}^{1}$, Lebesgue's dominated convergence theorem implies that

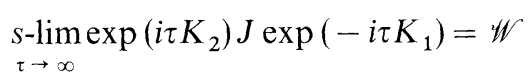

exists on $\mathfrak{R}_{2}$.

Statement (3) is a direct consequence of statements (1) and (2). Finally we prove statement (4). Let the operators $T$ on $\Re_{2}, O$ on $\Omega_{1}$ and $\bar{W}$ from $\Re_{1}$ to $\Re_{2}$ be defined as

$$
\begin{aligned}
& (T u)(t)=U(t, 0) u(t), \quad u \in \mathfrak{S}_{2} ; \\
& (O \tilde{u})(t)={ }^{\dagger}\left(e^{i t H_{0}} u(t), e^{i t \mu_{1}}{ }^{1} \sigma_{1,1}(t) \ldots, e^{i t \mu_{N, m N}} \sigma_{N, m_{N}}(t)\right) ; \\
& \left(\overline{\mathscr{W}}^{u}\right)(t)=W_{0}(0) u(t) \oplus\left(\sum_{j, k} \sigma_{j, k}(t) \otimes W_{j}(0) \phi_{j, k}\right) .
\end{aligned}
$$

$T$ is unitary on $\Re_{2}$ and $O$ is unitary on $\Re_{1}$. By (3.14) we have $\mathscr{W}=T \overline{\mathscr{W}} O$, hence $R(\mathscr{W})=T R(\bar{W})$ and if $R(\mathscr{W})=\mathfrak{\Omega}_{2}, R(\bar{W})=\mathfrak{S}_{2}$. By asymptotic orthogonality,

$$
\begin{aligned}
R(\bar{W}) & =\left(L^{2}\left(\mathbb{R}^{1}\right) \otimes R\left(W_{0}(0)\right)\right) \oplus\left(\sum_{j, k} \oplus\left(L^{2}\left(\mathbb{R}^{1}\right) \otimes\left\langle W_{j}(0) \phi_{j, k}\right\rangle\right)\right. \\
& =L^{2}\left(\mathbb{R}^{1}\right) \otimes\left(R\left(W_{0}(0)\right) \oplus \sum_{j, k} \oplus\left\langle W_{j}(0) \phi_{j, k}\right\rangle\right),
\end{aligned}
$$

where $\left\langle W_{j}(0) \phi_{j, k}\right\rangle$ stands for the one dimentional subspace spanned by 


$$
\begin{aligned}
& W_{j}(0) \phi_{j, k} \text {. Hence if } R(\overline{\mathscr{W}})=\mathfrak{R}_{2}=L^{2}\left(\mathbb{R}^{1}\right) \otimes L^{2}\left(\mathbb{R}^{n}\right), \\
& \mathfrak{H}=R\left(W_{0}(0)\right) \oplus \sum_{j, k} \oplus\left\langle W_{j}(0) \phi_{j, k}\right\rangle,
\end{aligned}
$$

which obviously implies statement (4).

\subsection{Abstract Stationary Theory}

By Lemma 3.1, the proof of the completeness of the wave operators is reduced to prove $R(\mathscr{W})=\Omega_{2}$. For proving this we shall apply the following abstract theorem due to Kato [8].

Theorem 3.2. (Kato $[8]$ ). For $j=1,2, K_{j}$ be a selfadjoint operator on the Hilbert space $\mathfrak{S}_{j}$ with the resolvent $R_{j}(z)=\left(K_{j}-z\right)^{-1}$ and the spectral measure $E_{j}(d \lambda)$. Let $J \in \mathbf{B}\left(\mathfrak{S}_{1}, \mathfrak{S}_{2}\right)$ be the identification operator and $I \subset \mathbb{B}^{1}$ be a Borel measurable subset of $\mathbb{R}^{1}$. Suppose the following conditions be satisfied.

(1) $\lim _{\tau \rightarrow+\infty}\left\|J \exp \left(-i \tau K_{1}\right) u\right\|=\|u\|, \quad u \in K_{1}$.

(2) There exists a linear manifold $\mathfrak{X}_{j}$ of $\Re_{j}$ such that there is no proper subspace of $\Omega_{j}$ invariant under $K_{j}$ and containing $\mathfrak{X}_{j}$ and such that $\mathfrak{X}_{j}$ is a normed space with its own norm (we write the completion of $\mathfrak{X}_{j}$ as $\overline{\mathfrak{X}}_{j}$ ) satisfying the following conditions: (2.i) For $x, y \in \mathfrak{X}_{1}, f_{0}(z, x, y)=\pi^{-1}|\operatorname{Im} z|\left(R_{1}(z) x, R_{1}(z) y\right)$ defined for $z \in \mathbb{C}_{ \pm}(I)$ has $a$ continuous boundary value for $z=\lambda \in I$.

(2ii) There is a strong continuous family of operators $Y(z) \in \mathbf{B}\left(\overline{\mathfrak{X}}_{2}, \overline{\mathfrak{X}}_{1}\right)$ defined for $z \in \mathbb{C}_{ \pm}(I) \cup I$ such that if $z \in \mathbb{C}_{ \pm}(I), Y(z)$ maps $\mathfrak{X}_{2}$ into $\mathfrak{X}_{1}$ with

$$
R_{2}(z) y=J R_{1}(z) Y(z) y, \quad \text { for } \quad y \in \mathfrak{X}_{2} .
$$

Then $K_{1}$ and $K_{2}$ are spectrally absolutely continuous on $I$ and there exists $Z_{ \pm} \in \mathbf{B}\left(\Re_{2}, \Re_{1}\right)$ which is partially isometric with initial set $E_{2}(I)$ and final set $E_{1}(I)$ and such that

$$
s \text {-Abel } \lim _{\tau \rightarrow \pm \infty} \exp \left(i \tau K_{2}\right) J \exp \left(-i \tau K_{1}\right) Z_{ \pm}=E_{2}(I)
$$

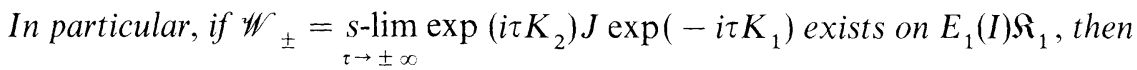

$$
R\left(\mathscr{W}_{ \pm}\right) \supset E_{2}(I) \Omega_{2}
$$

\subsection{The Faddeev's Matrix}

In the following subsections we shall prove that the conditions of Theorem 3.2 are satisfied for our operators $K_{1}$ and $K_{2}$, taking the spaces $\mathfrak{X}_{1}, \mathfrak{X}_{2}$ and the set $I$ appropriately. In this subsection, we shall derive the decomposition formula (3.15) for the resolvent $R_{2}(z)=\left(K_{2}-z\right)^{-1}$, postponing the proof of various estimates necessary for its justification until next subsection, although we shall prove some 
preliminary lemmas here. To avoid unnecessary complexity, we assume here and hereafter that $m_{j}=1$ for $j=1,2, \ldots, N$ and write $\mu_{j, k}, \phi_{j, k}$ and $L_{j, k}$ as $\mu_{j}, \phi_{j}$ and $L_{j}$ simply. The general case can be treated by a simple modification of the formulas which will appear in what follows.

We set $\Re_{3}=\bigoplus_{j=1}^{N}\left(L^{2}\left(\mathbb{R}^{1}\right) \otimes L^{2}\left(\mathbb{R}^{n}\right)\right)$ and define the operators $\mathscr{A}$ and $\mathscr{B}$ from $L^{2}\left(\mathbb{R}^{1}\right) \otimes L^{2}\left(\mathbb{R}^{n}\right)$ to $\Re_{3}$ as

$$
\left\{\begin{array}{l}
\mathscr{A} u={ }^{\dagger}\left(\mathscr{A}_{1} u(t, x), \ldots, \mathscr{A}_{N} u(t, x)\right), \\
\mathscr{B} u={ }^{\dagger}\left(\mathscr{B}_{1} u(t, x), \ldots, \mathscr{B}_{N} u(t, x)\right),
\end{array}\right.
$$

where

$$
\begin{aligned}
& \mathscr{A}_{j} u(t, x)=A_{j}\left(x-v_{j} t\right) u(t, x), \\
& \mathscr{B}_{j} u(t, x)=B_{j}\left(x-v_{j} t\right) u(t, x) .
\end{aligned}
$$

We write $G_{j}(z)=\left(L_{j}-z\right)^{-1}, j=0,1, \ldots, N$.

Lemma 3.3. Let $\mathscr{M}(z)$ be any one of $\mathscr{A} G_{0}(z) \mathscr{B}^{*}, \mathscr{B} G_{0}(z) \mathscr{A}^{*}, \mathscr{A} G_{0}(z) \mathscr{A}^{*}$, $\mathscr{B} G_{0}(z) \mathscr{B}^{*}$. Then

(1) $\mathscr{M}(z)$ is a $\mathbf{B}\left(\Omega_{2}\right)$-valued analytic function of $z \in \mathbb{C}_{ \pm}$and is uniformly bounded there.

(2) $\mathscr{M}(z)$ can be extended to the closed cut plane as a $\mathbf{B}\left(\Omega_{3}\right)$-valued Hölder continuous function of $z$.

(3) $\|\mathscr{M}(z)\| \rightarrow 0$ as $|\operatorname{Im} z| \rightarrow \infty$.

In particular $\mathscr{A}$ and $\mathscr{B}$ are $L_{0}$-smooth in the sense of Kato [7]. Let us write as $\mathscr{M}(\lambda \pm i 0)$ $\left(\lambda \in \mathbb{R}^{1}\right)$ the boundary values on the reals.

Proof. Let us write the generic element of $\Omega_{3}$ as $u=\left(u_{1}, \ldots, u_{N}\right)$. By the definition, for $\operatorname{Im} z>0$ (we prove only this case, the other case can be proved similarly),

$$
\begin{aligned}
& \left(\mathscr{A} G_{0}(z) \mathscr{B}^{*} u\right)_{j}(t) \\
& \quad=\sum_{k=1}^{N} i \int_{0}^{\infty} e^{i s z} A_{j}\left(x-v_{j} t\right) e^{-i s H_{0}} B_{k}\left(x-v_{k}(t-s)\right) u_{k}(t-s) d s .
\end{aligned}
$$

Therefore taking $q \geqq 1$ as $n / \delta<q<n$, we have by Lemma 2.2, (1),

$$
\begin{aligned}
& \left\|\left(\mathscr{A} G_{0}(z) \mathscr{B} * u\right)_{j}(t)\right\| \\
& \quad \leqq C \sum_{k=1}^{N} \int_{0}^{\infty} e^{-\operatorname{sIm} z} \min _{p, q}\left(\left\|A_{j}\right\|_{p}\left\|B_{k}\right\|_{p}|s|^{-n / p},\left\|A_{j}\right\|_{q}\left\|B_{k}\right\|_{q}|s|^{-n / q}\right)\left\|u_{k}(t-s)\right\| d s .
\end{aligned}
$$

Here in this section $\|f\|_{p}$ 's are $L^{p}$-norm of $f$. For $\operatorname{Im} z \geqq 0$,

$$
C_{j, k}(z)=\int_{0}^{\infty} e^{-s \operatorname{Im} z} \min _{p, q}\left(\left\|A_{j}\right\|_{p}\left\|B_{k}\right\|_{p} s^{-n / p},\left\|A_{j}\right\|_{q}\left\|B_{k}\right\|_{q} s^{-n / q}\right) d s
$$

is uniformly bounded and

$$
C_{j, k}(z) \rightarrow 0 \text { as } \operatorname{Im} z \rightarrow \infty .
$$


Young's inequality shows that

$$
\left\|\left(\mathscr{A} G_{0}(z) \mathscr{B}^{*} u\right)_{j}\right\|_{\Omega_{2}} \leqq \sum_{k=1}^{N} C_{j, k}(z)\left\|u_{k}\right\|_{\Omega_{2}} .
$$

Hence by Schwartz's inequality we get

$$
\left\|\mathscr{A} G_{0}(z) \mathscr{B} * u\right\|_{\mathfrak{S}_{3}} \leqq\left(\sum_{j, k} C_{j, k}(z)^{2}\right)^{1 / 2}\|u\|_{\Omega_{3}} .
$$

By (3.22) and (3.25), we see that $\mathscr{A} G_{0}(z) \mathscr{B}^{*}$ has the bounded closed extension $\left[\mathscr{A} G_{0}(z) \mathscr{B}^{*}\right]$ and this is obviously a $\mathbf{B}\left(\Re_{3}\right)$-valued analytic function of $z \in \mathbb{C}_{+}$. By (3.23) and (3.25), we also see that

$$
\lim _{\operatorname{Im} z \rightarrow \infty}\left\|\mathscr{A} G_{0}(z) \mathscr{B}^{*}\right\|=0 .
$$

For proving that $\mathscr{A} G_{0}(z) \mathscr{B}^{*}$ can be extended to $\mathbb{C}_{+} \cup \mathbb{R}^{1}$ as a $\mathbf{B}\left(\Omega_{3}\right)$-valued Hölder continuous function, it suffices to prove that $\mathscr{A} G_{0}(z) \mathscr{B}^{*}$ is uniformly Hölder continuous on $\mathbb{C}_{+}$. We first note that if $\operatorname{Im} z, \operatorname{Im} z^{\prime}>0$ and $s>0$,

$$
\left|e^{i s z}-e^{i s z^{\prime}}\right| \leqq s^{\alpha}\left|z-z^{\prime}\right|^{\alpha}
$$

for any $0 \leqq \alpha \leqq 1$. Hence by (3.20) and Lemma 2.2, (1),

$$
\begin{aligned}
& \left\|\left(\mathscr{A} G_{0}(z) \mathscr{B}^{*} u\right)_{j}(t)-\left(\mathscr{A} G_{0}\left(z^{\prime}\right) \mathscr{B}^{*} u\right)_{j}(t)\right\| \\
& \quad \leqq \sum_{k=1}^{N} \int_{0}^{\infty} s^{\alpha}\left|z-z^{\prime}\right|^{\alpha} \min _{p, q}\left(\left\|A_{j}\right\| p\left\|B_{k}\right\|_{p} s^{-n / p},\left\|A_{j}\right\|_{q}\left\|B_{k}\right\|_{q} s^{-n / q}\right)\left\|u_{k}(t-s)\right\| d s .
\end{aligned}
$$

By taking $0<\alpha<(n / q)-1$, we see that

$$
C_{j, k}^{\alpha} \equiv \int_{0}^{\infty} s^{\alpha} \min \left(\left\|A_{j}\right\|_{p}\left\|B_{k}\right\|_{p} s^{-n / p},\left\|A_{j}\right\|_{q}\left\|B_{k}\right\|_{q} s^{-n / q}\right) d s<\infty .
$$

Hence by Young's and Schwartz's inequalities we get

$$
\begin{aligned}
& \left\|\mathscr{A} G_{0}(z) \mathscr{B}^{*} u-\mathscr{A} G_{0}\left(z^{\prime}\right) \mathscr{B}^{*} u\right\|_{\Omega_{3}} \\
& \quad \leqq\left|z-z^{\prime}\right|^{\alpha}\left(\sum_{j, k}\left(C_{j, k}^{\alpha}\right)^{2}\right)^{1 / 2}\|u\|_{\Omega_{3} .}
\end{aligned}
$$

Thus we see that $\mathscr{A} G_{0}(z) \mathscr{B}^{*}$ is uniformly Hölder continuous on $\mathbb{C}_{+}$with exponent $\alpha$, $\alpha$ is an arbitrary number satisfying $0<\alpha<(n / q)-1, \alpha \leqq 1$. This completes the proof of the statements (1)-(3) for $\mathscr{A} G_{0}(z) \mathscr{B}^{*}$. The forgoing arguments obviously apply for any other operator being considered here. The $L_{0}$-smoothness of $\mathscr{A}$ (or $\mathscr{B}$ ) is obvious by the statement (1) for $\mathscr{A} G_{0}(z) \mathscr{A}^{*}$ (or $\left.\mathscr{B} G_{0}(z) \mathscr{B}^{*}\right)$ and the definition of the $L_{0}$-smoothness (see Reed-Simon [13], Theorem XIII. 25).

(Q.E.D.)

Remark. By (3.21) we have

$$
C_{j, k}(z) \leqq C_{p, q} \max _{p, q}\left(\left\|A_{j}\right\|_{p}\left\|B_{k}\right\|_{p^{\prime}}\left\|A_{j}\right\|_{q}\left\|B_{k}\right\|_{q}\right)
$$


where $C_{p, q}$ is a constant depending only on $p$ and $q$. Hence

$$
\left\|\mathscr{A} G_{0}(z) \mathscr{B}^{*}\right\| \leqq C_{p, q} \max _{p, q}\left(\left\|A_{j}\right\|_{p}\left\|B_{k}\right\|_{p^{\prime}}\left\|A_{j}\right\|_{q}\left\|B_{q}\right\|_{q}: j, k=1, \ldots, N\right) \text {. }
$$

Therefore if all $A_{j}$ 's (or $B_{k}$ 's) converge to zero in $L^{p}\left(\mathbb{R}^{n}\right)$ and $L^{q}\left(\mathbb{R}^{n}\right)(1 \leqq q<n / 2<p$ $\leqq \infty)$, then the operator $\mathscr{A} G_{0}(z) \mathscr{B}^{*}$ converges to zero in operator norm. Similar result obviously holds for other operators being considered in Lemma 3.3. This result will be used in the proof of Lemma 3.8.

We need the following auxiliary operators.

For $j=1,2, \ldots, N$, define one parameter unitary group $\mathscr{V}_{j}(\tau)$ on $\mathfrak{S}_{2}$ as

$$
\left(\mathscr{V}_{j}(\tau) f\right)(t)=U_{j}(t, t-\tau) f(t-\tau)
$$

and write the generator of this group as $\mathscr{L}_{j}$ :

$$
\mathscr{V}_{j}(\tau)=e^{-i \tau \mathscr{L}_{j}}, \quad-\infty<\tau<\infty .
$$

We write as

$$
\mathscr{G}_{j}(z)=\left(\mathscr{L}_{j}-z\right)^{-1}, \quad \operatorname{Im} z \neq 0 .
$$

Lemma 3.4. For $\operatorname{Im} z \neq 0, j=1, \ldots, N$,

$$
\begin{aligned}
& R_{2}(z)=G_{0}(z)-\left[\mathscr{B} G_{0}(\bar{z})\right]^{*}\left(1+\mathscr{Q}_{0}(z)\right)^{-1} \mathscr{A} G_{0}(z), \\
& \mathscr{G}_{j}(z)=G_{0}(z)-\left[\mathscr{B}_{j} G_{0}(\bar{z})\right]^{*}\left(1+\mathscr{Q}_{j}(z)\right)^{-1} \mathscr{A}_{j} G_{0}(z),
\end{aligned}
$$

where $\mathscr{2}_{0}(z)=\mathscr{A} G_{0}(z) \mathscr{B}^{*}$ and $\mathscr{2}_{j}=\mathscr{A}_{j} G_{0}(z) \mathscr{B}_{j}^{*}$.

This lemma can be proved similarly as Lemma 3.3 of Yajima [16], using Lemma 3.3 above and (B) of Sect. 1. Hence the proof is omitted here.

Now we proceed to the derivation of (3.15). Let

$D(z)=(N \times N)$-diagonal matrix with $(j, j)$-element $\mathscr{A}_{j} G_{0}(z) \mathscr{B}_{j}^{*}$.

$$
F_{0}(z)=Q(z)-D(z) \text {. }
$$

By (3.32) we have

$$
\begin{aligned}
& (1+D(z))^{-1}=(N \times N) \text {-diagonal matrix with }(j, j) \text {-element } \\
& \quad 1-\mathscr{A}_{j} \mathscr{G}_{j}(z) \mathscr{B}_{j}^{*} ; \\
& (1+D(z))^{-1} \mathscr{A} G_{0}(z)={ }^{\dagger}\left(\mathscr{A}_{1} \mathscr{G}_{1}(z), \ldots, \mathscr{A}_{N} \mathscr{G}_{N}(z)\right) ; \\
& (1+D(z))^{-1} F_{0}(z)=(N \times N) \text {-matrix with } \\
& \quad(j, k) \text {-element } \widehat{\delta}_{j, k} \mathscr{A}_{j} \mathscr{G}_{j}(z) \mathscr{B}_{k},
\end{aligned}
$$

where

$$
\widehat{\delta}_{j, k}= \begin{cases}1 & \text { if } j \neq k \\ 0 & \text { if } j=k .\end{cases}
$$

We write the R.H.S. of (3.34) and (3.35) as

$$
\begin{aligned}
\hat{A} \hat{G}(z) & ={ }^{\dagger}\left(\mathscr{A}_{1} \mathscr{G}_{1}(z), \ldots, \mathscr{A}_{N} \mathscr{G}_{N}(z)\right), \\
F(z) & =\left(\hat{\delta}_{j, k} \mathscr{A}_{j} \mathscr{G}_{j}(z) \mathscr{B}_{k}\right)_{j, k} .
\end{aligned}
$$


Thus combining (3.33)-(3.38) with (3.31), we have

$$
\begin{aligned}
& R_{2}(z) \\
& \quad=G_{0}(z)-\left[\mathscr{B} G_{0}(\bar{z})\right]^{*}\left(1+(1+D(z))^{-1} F_{0}(z)\right)^{-1}(1+D(z))^{-1} \mathscr{A} G_{0}(z) \\
& \quad=G_{0}(z)-\left[\mathscr{B} G_{0}(\bar{z})\right]^{*}(1+F(z))^{-1} \hat{A} \hat{G}(z) .
\end{aligned}
$$

To go further we need the following lemma.

\section{Lemma 3.5.}

$$
T_{v_{j}}\left(-i \partial / \partial t \otimes I+I \otimes H_{j}-z\right)^{-1} T_{v_{j}}^{*}=\mathscr{G}_{j}(z) .
$$

Proof. Note that

$$
\left(T_{v_{j}}^{*} f\right)(t, x)=e^{-i x \cdot v_{j}-i v_{j}^{2} t / 2} f\left(t, x+v_{j} t\right) .
$$

Then simple calculations show

$$
\begin{aligned}
& T_{v_{j}} G_{0}(z) T_{v_{j}}^{*}=G_{0}(z), \\
& T_{v_{j}}^{*} \mathscr{A}_{j} T_{v_{j}}=I \otimes A_{j}, \quad T_{v_{j}}^{*} \mathscr{B}_{j}^{*} T_{v_{j}}=I \otimes B_{j}^{*} .
\end{aligned}
$$

We apply to the both sides of (3.32), $T_{v_{j}}$ from the right and $T_{v_{j}}^{*}$ from the left. Then we get by (3.42) and (3.43) that

$$
\begin{aligned}
T_{v_{j}}^{*} \mathscr{G}_{j}(z) T_{v_{j}}= & T_{v_{j}}^{*} G_{0}(z) T_{v_{j}}-\left[T_{v_{j}}^{*} \mathscr{B}_{j} T_{v_{j}}\left(T_{v_{j}}^{*} G_{0}(\bar{z}) T_{v_{j}}\right)\right]^{*} \\
& \times\left(1+T_{v_{j}}^{*} \mathscr{A}_{j} T_{v_{j}}\left(T_{v_{j}}^{*} G_{0}(z) T_{v_{j}}\right)\left(T_{v_{j}}^{*} \mathscr{B}_{j} T_{v_{j}}\right)\right)^{-1} \\
& \times T_{v_{j}}^{*} \mathscr{A}_{j} T_{v_{j}}\left(T_{v_{j}}^{*} G_{0}(z) T_{v_{j}}\right) \\
= & G_{0}(z)-\left[\left(I \otimes B_{j}\right) G_{0}(\bar{z})\right]^{*}\left(1+\left(I \otimes A_{j}\right) G_{0}(z)\left(I \otimes B_{j}\right)\right)^{-1} \\
& \times\left(I \otimes A_{j}\right) G_{0}(z) .
\end{aligned}
$$

We regard as $L^{2}\left(\mathbb{R}^{1}\right) \otimes L^{2}\left(\mathbb{R}^{n}\right)=\int^{\oplus} L^{2}\left(\mathbb{R}^{n}\right) d t$. Then the last member of (3.44) is written as $\quad \mathscr{F}_{t}^{-1} \int^{\oplus}\left(r_{0}(z-t)-\left[r_{0}(z-\tau) B_{j}\right]\left(1+Q_{j}(z-\tau)\right)^{-1}\left[A r_{0}(z-\tau)\right]\right) d \tau \mathscr{F}_{t}=\mathscr{F}_{t}^{-1} \times$ $\int^{\oplus}\left(H_{j}-z+\tau\right)^{-1} d \tau \mathscr{F}_{t}=\left(-i \partial / \partial t \otimes I+I \otimes H_{j}-z\right)^{-1}$. This proves (3.40). (Q.E.D.)

By spectral decomposition we have

$$
H_{j}=H_{j}\left(1-P_{j}\right)+\mu_{j} P_{j} \equiv H_{j}^{c}+\mu_{j} P_{j} .
$$

We write as $1-P_{j}=P_{j}^{c}$. Then

$$
\begin{aligned}
& \left(-i \partial / \partial t \otimes I+I \otimes H_{j}-z\right)^{-1} \\
& \quad=\left(-i \partial / \partial t \otimes I+I \otimes H_{j}^{c}-z\right)^{-1}\left(I \otimes P_{j}^{c}\right)+G_{j}(z) \otimes P_{j} .
\end{aligned}
$$

We write

$$
T_{v_{j}}\left(-i \partial / \partial t \otimes I+I \otimes H_{j}^{c}-z\right)^{-1}\left(I \otimes P_{j}^{c}\right) T_{v_{j}}^{*}=\mathscr{G}_{j}^{c}(z)
$$

and set $\Gamma_{j} \in \mathbf{B}\left(L^{2}\left(\mathbb{R}^{1}\right) \otimes L^{2}\left(\mathbb{R}^{n}\right), L^{2}\left(\mathbb{R}^{1}\right)\right)$ as

$\left(\Gamma_{j} u\right)(t)=\int \phi_{j}(x) u(t, x) d x$. 
Then equations (3.40), (3.45) and (3.46) imply

$$
\mathscr{G}_{j}(z)=\mathscr{G}_{j}^{c}(z)+T_{v_{j}} \Gamma_{j}^{*} G_{j}(z) \Gamma_{j} T_{v_{j}}^{*} \text {. }
$$

Now we proceed to further decomposition of (3.39). We define $(1 \times 2 N)$-matrix $L(z),(N \times 2 N)$-matrix $X(z)$ and $(2 N \times 2 N)$-matrix $A(z)$ as follows:

$$
\begin{aligned}
& L(z)={ }^{\dagger}\left(\mathscr{A}_{1} \mathscr{G}_{1}^{c}(z), \Gamma_{1} T_{v_{1}}^{*}, \ldots, \mathscr{A}_{N} \mathscr{G}_{N}^{c}(z), \Gamma_{N} T_{v_{N}}^{*}\right) \\
& X(z)=\left(X_{j k}(z)\right)_{j=1, \ldots, N: k=1, \ldots, 2 N,} \\
& X_{j k}(z)= \begin{cases}I, \quad k=2 j-1, \quad j=1, \ldots, N, \\
\mathscr{A}_{j} T_{v_{j}} \Gamma_{j}^{*} G_{j}(z), \quad k=2 j, \quad j=1, \ldots, N, \\
0, \quad \text { otherwise; }\end{cases} \\
& A(z)=\left(A_{j k}(z)\right)_{j, k}=i, \ldots, 2 N, \\
& A_{j k}(z)= \begin{cases}\mathscr{A}_{m} \mathscr{G}_{m}^{c}(z) \mathscr{B}_{l}^{*}, & j=2 m-1, \quad k=2 l-1, \quad m \neq l, \\
\mathscr{A}_{m} \mathscr{G}_{m}^{c}(z) \mathscr{B}_{l}^{*} \mathscr{A}_{l} T_{v l} \Gamma_{l}^{*} G_{l}(z), \quad j=2 m-1, \quad k=2 l, \quad m \neq l . \\
\Gamma_{m} T_{v_{m}}^{*} \mathscr{B}_{l}^{*} & j=2 m, \quad k=2 l-1, \quad m \neq l . \\
\Gamma_{m} T_{v_{m}}^{*} \mathscr{B}_{l}^{*} \mathscr{A}_{l} T_{v_{l}} \Gamma_{l}^{*} G_{l}(z), & j=2 m, \quad k=2 l, \quad m \neq l . \\
0, & \text { otherwise. }\end{cases}
\end{aligned}
$$

$A(z)$ is a substitute of so-called Faddeev matrix in three body problem. By (3.32) and (3.47), we can easily see that

$$
\begin{aligned}
& \hat{A} \widehat{G}(z)=X(z) L(z) ; \\
& F(z) X(z)=X(z) A(z) ;
\end{aligned}
$$

and hence

$$
(1+F(z))^{-1} X(z)=X(z)(1+A(z))^{-1} .
$$

Here the existence of the inverse in both side of (3.53) can be proved by the standard way (Faddeev [3], p. 50, Lemma 7.5). Combining (3.39) with (3.51) and (3.53), we get

$$
R_{2}(z)=G_{0}(z)-\left[\mathscr{B} G_{0}(\bar{z})\right]^{*} X(z)(1+A(z))^{-1} L(z) .
$$

By definition,

$$
\begin{aligned}
& {\left[\mathscr{B}_{0}(\bar{z})\right]^{*} X(z)} \\
& \quad={ }^{\dagger}\left(G_{0}(z) \mathscr{B}_{1}, G_{0}(z) \hat{V}_{1} T_{v_{1}} \Gamma_{1}^{*} G_{1}(z), \ldots, G_{0}(z) \mathscr{B}_{N}, G_{0}(z) \hat{V}_{N} T_{v_{N}} \Gamma_{N}^{*} G_{N}(z)\right) .
\end{aligned}
$$

On the other hand by (3.32), (3.40) and (3.44),

$$
\begin{aligned}
G_{0}(z) \hat{V}_{j} T_{v_{j}} \Gamma_{j}^{*} G_{j}(z) & =G_{0}(z) \hat{V}_{j} \mathscr{G}_{j}(z) T_{v_{j}} \Gamma_{j}^{*} \\
& =\left(G_{0}(z)-G_{j}(z)\right) T_{v_{j}} \Gamma_{j}^{*} \\
& =G_{0}(z) T_{v_{j}} \Gamma_{j}^{*}-T_{v_{j}} \Gamma_{j}^{*} G_{j}(z) .
\end{aligned}
$$

Here in (3.55) and (3.56) we wrote as $\hat{V}_{j}=\mathscr{A}_{j} \mathscr{B}_{j}^{*}$ simply. We write as

$$
(1+A(z))^{-1} L(z)={ }^{\dagger}\left(\mathscr{H}_{1}(z), \hbar_{1}\left(z_{1}\right), \ldots, \mathscr{H}_{N}(z), \hbar_{N}(z)\right)
$$




$$
\begin{aligned}
& Y_{0}(z)=1-\sum_{j=1}^{N}\left(\mathscr{B}_{j} \mathscr{H}_{j}(z)+T_{v_{j}} \Gamma_{j}^{*} h_{j}(z)\right) ; \\
& Y_{j}(z)=-h_{j}(z) \quad(j=1,2, \ldots, N) .
\end{aligned}
$$

Then combining (3.54)-(3.59), we finally get

$$
\begin{aligned}
R_{2}(z) & =G_{0}(z)\left(1-\sum_{j=1}^{N}\left(\mathscr{B}_{j} \mathscr{H}_{j}(z)+T_{v_{j}} \Gamma_{j}^{*} h_{j}(z)\right)\right)-\sum_{j=1}^{N} T_{v_{j}} \Gamma_{j}^{*} G_{j}(z) h_{j}(z) \\
& =G_{0}(z) Y_{0}(z)+\sum_{j=1}^{N} T_{v_{j}}\left(G_{j}(z) Y_{j}(z) \otimes \phi_{j}\right) \\
& =J R_{1}(z) Y(z)
\end{aligned}
$$

where, of course,

$$
Y(z)={ }^{\dagger}\left(Y_{0}(z), Y_{1}(z), \ldots, Y_{N}(z)\right)
$$

\subsection{Estimates of Operators}

In this subsection we give several estimates of the operators which are necersary for the application of Theorem 3.2. To start with, we prove the following lemma.

Lemma 3.6. Let $\gamma>1 / 2$ and $j=1,2, \ldots, N$. Then $G_{j}(z)$ satisfies the following properties.

(1) $G_{j}(z)$ is a $\mathbf{B}\left(L_{\gamma}^{2}\left(\mathbb{R}^{1}\right), L_{-\gamma}^{2}\left(\mathbb{R}^{1}\right)\right)$-valued uniformly bounded analytic function of $z \in \mathbb{C}_{ \pm}$and can be extended to $\mathbb{C}_{ \pm} \cup \mathbb{R}^{1}$ as a Hölder continuous function.

(2) $G_{j}(z) \in \mathbf{B}_{\infty}\left(L_{\gamma}^{2}\left(\mathbb{R}^{1}\right), L_{-\gamma}^{2}\left(\mathbb{R}^{1}\right)\right)$.

(3) $\left\|G_{j}(z)\right\|_{\mathbf{B}_{\left(L_{\gamma}^{2}, L_{-\gamma}^{2}\right)} \rightarrow 0}$ as $\quad|\operatorname{Im} z| \rightarrow \infty$.

Proof. Let $m(t)=\left(1+t^{2}\right)^{-\gamma / 2}$ and $M$ be the multiplication operator by $m(t)$. Then $M$ is a unitary operator from $L^{2}\left(\mathbb{R}^{1}\right)$ to $L_{\gamma}^{2}\left(\mathbb{R}^{1}\right)$ as well as from $L_{-\gamma}^{2}\left(\mathbb{R}^{1}\right)$ to $L^{2}\left(\mathbb{R}^{1}\right)$. Hence for proving (1)-(3), it suffices to prove that

$(1)^{\prime} G_{j}^{\prime}(z)=M G_{j}(z) M$ is $\mathbf{B}\left(L^{2}\left(\mathbb{R}^{1}\right)\right)$-valued uniformly bounded analytic function of $z \in \mathbb{C}_{ \pm}$and can be extended to $\mathbb{C}_{ \pm} \cup \mathbb{R}^{1}$ as a Hölder continuous function;

$(2)^{\prime} G_{j}^{\prime}(z) \in \mathbf{B}_{\infty}\left(L^{2}\left(\mathbb{R}^{1}\right)\right)$;

$(3)^{\prime}\left\|G_{j}^{\prime}(z)\right\|_{\mathbf{B}\left(L^{2}\left(\mathbb{R}^{1}\right)\right)} \rightarrow 0$ as $|\operatorname{Im} z| \rightarrow \infty$.

We prove $(1)^{\prime}-(3)^{\prime}$ for $z \in \mathbb{C}_{+}$. The case $z \in \mathbb{C}_{-}$can be proved similarly. Since $G_{j}(z)$ is a convolution operator with the function $i \exp \left(i\left(z-\mu_{j}\right) t\right) \theta(t), \theta(t)$ is the Heaviside function, $G_{j}^{\prime}(z)$ is the integral operator with the kernel

$$
\begin{aligned}
& K_{j, \gamma}(t, s ; z)=i\left(1+t^{2}\right)^{-\gamma / 2} e^{i\left(z-\mu_{j}\right)(t-s)} \theta(t-s)\left(1+s^{2}\right)^{-\gamma / 2} . \\
& \int\left|K_{j, \gamma}(t, s ; z)\right|^{2} d t d s \\
& \quad \leqq \int\left(1+t^{2}\right)^{-\gamma}\left(1+s^{2}\right)^{-\gamma} e^{-\operatorname{Im} z|t-s|} d t d s .
\end{aligned}
$$

The R.H.S. of (3.62) is uniformly bounded for $z \in \mathbb{C}_{+}$and converges to zero as $\operatorname{Im} z \rightarrow \infty$, since $\gamma>1 / 2$. Hence $G_{j}^{\prime}(z)$ is a Hilbert-Schimdt operator on $L^{2}\left(\mathbb{R}^{1}\right)$ and 
the Hilbert-Schmidt norm converges to zero as $\operatorname{Im} z \rightarrow \infty$. This proves (2)' and (3)' and a part of $(1)^{\prime}$. Thus for completing the proof it suffices to prove that $G_{j}^{\prime}(z)$ is a uniformly Hölder continuous $\mathbf{B}\left(L^{2}\left(\mathbb{R}^{1}\right)\right)$-valued function. This is simple, since for any $0<\alpha<2 \gamma-1$ and $\alpha \leqq 1$,

$$
\begin{aligned}
& \int\left|K_{j, \gamma}(t, s ; z)-K_{j, \gamma}\left(t, s ; z^{\prime}\right)\right| d t d s \\
& \quad \leqq\left|z-z^{\prime}\right|^{\alpha} \int|t-s|^{\alpha}\left(1+t^{2}\right)^{-\gamma}\left(1+s^{2}\right)^{-\gamma} d t d s
\end{aligned}
$$

and the integral in the R.H.S. is finite.

We define the spaces $\mathfrak{X}_{1}$ and $\mathfrak{X}_{2}$ as follows:

$$
\left\{\begin{array}{l}
\mathfrak{X}_{1}=R\left(\mathscr{B}^{*}\right) \oplus \sum_{j=1}^{N} L_{\delta}^{2}\left(\mathbb{R}^{1}\right) ; \\
\mathfrak{X}_{2}=L_{\delta}^{2}\left(\mathbb{R}^{n+1}\right) .
\end{array}\right.
$$

$\mathfrak{X}_{1}$ and $\mathfrak{X}_{2}$ are equipped with the natural Hilbert space structure. Obviously $\mathfrak{X}_{1}$ and $\mathfrak{X}_{2}$ are dense linear submanifolds of $\Omega_{1}$ and $\Omega_{2}$, respectively.

Lemma 3.7. $\mathfrak{X}_{1}$ and $K_{1}$ satisfy the condition (2.i) of Theorem 3.2.

Proof. Since $\quad R_{1}(z) \tilde{u}=\left(K_{1}-z\right)^{-1} \tilde{u}={ }^{\dagger}\left(G_{0}(z) u, G_{1}(z) \sigma_{1}, \ldots, G_{N}(z) \sigma_{N}\right) \quad$ and $f_{0}(z, x, y)=|\operatorname{Im} z|\left(R_{1}(z) x, R_{1}(z) y\right)=(1 / 2 \pi i)\left(\left(R_{1}(z)-R_{1}(\bar{z})\right) x, y\right)$, it suffices to prove that $\mathscr{B}^{*} G_{0}(z) \mathscr{B}$ (or $G_{j}(z)$ ) can be extended to the closed cut plane $\mathbb{C}_{ \pm} \cup \mathbb{R}^{1}$ as a $\mathbf{B}\left(L^{2}\left(\mathbb{R}^{n+1}\right)\right)$ (or $\left.\mathbf{B}\left(L_{\delta}^{2}\left(\mathbb{R}^{1}\right), L_{-\delta}^{2}\left(\mathbb{R}^{1}\right)\right)\right)$-valued continuous function of $z$. For $G_{j}(z)(j=1,2, \ldots, N)$ this is proved in Lemma 3.6, and for $\mathscr{B}^{*} G_{0}(z) \mathscr{B}$ in Lemma 3.3.

(Q.E.D.)

The following is the key lemma in this section.

Lemma 3.8. If $j \neq k, \mathscr{A}_{j} G_{0}(z) \mathscr{B}_{k}^{*}$ is a compact operator on $L^{2}\left(\mathbb{R}^{n+1}\right)$ for any $z$ in the closed cut plane $\mathbb{C}_{ \pm} \cup \mathbb{R}^{1}$.

For proving the lemma we need the following preliminary lemmas. In Lemmas 3.9, 3.10 and Corollary $3.11, n$ is any positive integer.

Lemma 3.9. Let $\sigma, \rho>0$. If $K \in \mathbf{B}\left(L^{2}\left(\mathbb{R}^{n}\right), L_{\rho}^{2}\left(\mathbb{R}^{n}\right)\right)$ and $K \in \mathbf{B}\left(L^{2}\left(\mathbb{R}^{n}\right), H^{\sigma}\left(\mathbb{R}^{n}\right)\right)$, then $K$ is a compact operator on $L^{2}\left(\mathbb{R}^{n}\right)$.

Proof. Let $R>0$ and $\chi_{R}$ be the characteristic function of the ball $\{|x| \leqq R\}$. Then $\chi_{R} K$ is a compact operator on $L^{2}\left(\mathbb{R}^{n}\right)$ by Rellich's compactness theorem. On the other hand,

$$
\begin{aligned}
& \left\|K u-\chi_{R} K u\right\| \leqq\left(\int_{|x| \geqq R}|K u(x)|^{2} d x\right)^{1 / 2} \\
& \quad \leqq\left(1+R^{2}\right)^{-\rho / 2}\|K u\|_{L_{\rho}^{2}} \leqq\left(1+R^{2}\right)^{-\rho / 2}\|K\|_{B\left(L^{2}, L_{\rho}^{2}\right)}\|u\| .
\end{aligned}
$$

Therefore as $R \rightarrow \infty,\left\|K-\chi_{R} K\right\| \rightarrow 0$. This proves Lemma 3.9.

(Q.E.D.)

Lemma 3.10. Let $x \in \mathbb{R}^{n}, y \in \mathbb{R}^{m}$ and $\xi, \eta$ be the conjugate variables of $x, y$ ( $m$ may be 
zero). Let $g(\xi, \eta)$ be a function such that for any multi-index $\alpha, \sup _{\xi, \eta}\left|(\partial / \partial \xi)^{\alpha} g(\xi, \eta)\right|$ $\leqq C_{\alpha}<\infty$. Let $G\left(D_{x}, D_{y}\right)$ be the operator defined as

$$
G\left(D_{x}, D_{y}\right)=\mathscr{F}_{(x, y)}^{-1} g(\xi, \eta) \mathscr{F}_{(x, y)} .
$$

Then for any $\rho \in \mathbb{R}^{1}$,

$$
\left\|\left(1+|x|^{2}\right)^{\rho} G\left(D_{x}, D_{y}\right)\left(1+|x|^{2}\right)^{-\rho}\right\|<\infty .
$$

Proof. Let $\rho \geqq 0$ be an integer. By Parseval's relation and Leibniz's rule we have

$$
\begin{aligned}
&\left\|\left(1+|x|^{2}\right)^{\rho} G\left(D_{x}, D_{y}\right)\left(1+|x|^{2}\right)^{-\rho} u(x, y)\right\| \\
&=\left\|\left(1-\Delta_{\xi}\right)^{\rho} g(\xi, \eta)\left(1-\Delta_{\xi}\right)^{-\rho} \hat{u}(\xi, \eta)\right\| \\
&=\| \sum_{0 \leq \sigma \leq \rho}\left(\begin{array}{c}
\rho \\
\sigma
\end{array}\right)(-1)^{\sigma} \sum_{0 \leqq \beta \leqq 2 \tilde{\sigma}}\left(\begin{array}{c}
2 \hat{\sigma} \\
\beta
\end{array}\right)(\partial / \partial \xi)^{2 \tilde{\sigma}-\beta} g(\xi, \eta)(\partial / \partial \xi)^{\beta} \\
& \cdot\left(1-\Delta_{\xi}\right)^{-\rho} \hat{u}(\xi, \eta) \| \\
& \leqq \sum_{0 \leq \sigma \leq \rho} \sum_{0 \leq \beta \leq 2 \tilde{\sigma}}\left(\begin{array}{c}
\rho \\
\sigma
\end{array}\right)\left(\begin{array}{c}
2 \tilde{\sigma} \\
\beta
\end{array}\right) C_{2 \tilde{\sigma}-\beta}\left\|(\partial / \partial \xi)^{\beta}\left(1-\Delta_{\xi}\right)^{-\rho} \hat{u}(\xi, \eta)\right\|,
\end{aligned}
$$

where $2 \bar{\sigma}=(2 \sigma, 2 \sigma, \ldots, 2 \sigma)$. Since $0 \leqq \sigma \leqq \rho, 0 \leqq \beta \leqq 2 \bar{\sigma}$,

$$
\left\|(\partial / \partial \xi)^{\beta}\left(1+\Delta_{\xi}\right)^{-\rho} \hat{u}(\xi, \eta)\right\|=\left\|x^{\beta}\left(1+x^{2}\right)^{-\rho} u(x, y)\right\| \leqq\|u(x, y)\| .
$$

Combining this with (3.65), we get (3.64) for $\rho$ non-negative integers. For general $\rho$ $\geqq 0,(3.64)$ is the consequence of the above case and the interpolation theorem (Lions-Magenes [19], p. 27). We now prove the case $\rho<0$. Let $u, h \in C_{0}^{\infty}\left(\mathbb{R}^{n}\right)$. Then the result for the case $\rho>0$ implies

$$
\begin{aligned}
& \left.\mid\left(\left(1+|\mathrm{x}|^{2}\right)^{\rho} G\left(D_{x}, D_{y}\right)\left(1+|x|^{2}\right)^{-\rho} u, h\right)\right\} \\
& \leqq\left|\left(u,\left(1+|x|^{2}\right)^{-\rho} \bar{G}\left(D_{x}, D_{y}\right)\left(1+|x|^{2}\right)^{\rho} h\right)\right| \leqq C\|u\|\|h\| .
\end{aligned}
$$

This obviously implies (3.64).

(Q.E.D.)

The Fourier transform of Lemma 3.10 implies the following

Corollary 3.11. Let $f \in C^{\infty}\left(\mathbb{R}^{n}\right)$ and its derivatives are all bounded functions. Then the multiplication operator by $f$ is a bounded operator on $H^{s}\left(\mathbb{R}^{n}\right)$ for any $s \in \mathbb{R}^{1}$

Proof of Lemma 3.9. (i) We first show that it suffices to prove that $A(x-v t)$ $G_{0}( \pm i) B(x)$ is a compact operator on $\Re_{2}$ when $A, B \in C_{0}^{\infty}\left(\mathbb{R}^{n}\right)$ and $v \neq 0$. Since $\mathscr{A}_{j} G_{0}(z) \mathscr{B}_{k}$ is a $\mathbf{B}\left(\Re_{2}\right)$-valued continuous function of $z \in \mathbb{C}_{ \pm} \cup \mathbb{R}^{1}$ by Lemma 3.3, it suffices to prove that $\mathscr{A}_{j} G_{0}( \pm i) \mathscr{B}_{k} \in \mathbf{B}_{\infty}\left(\Omega_{2}\right)$. Choose $\omega(x) \in C_{0}^{\infty}\left(\mathbb{R}^{n}\right)$ such that $\omega(x) \geqq 0, \omega(x) \equiv 1$ near $x=0$ and $\int \omega(x) d x=1$. For $\varepsilon>0, \omega_{\varepsilon}(x)=\varepsilon^{-n} \omega(x / \varepsilon)$. Set $A_{j, \varepsilon}(x)=\omega(\varepsilon x)\left(A_{j} * \omega_{\varepsilon}\right)(x) \quad$ and $\quad B_{k, \varepsilon}(x)=\omega(\varepsilon x)\left(B_{k} * \omega_{\varepsilon}\right)(x)$. Then $A_{j, \varepsilon}$, $B_{k, \varepsilon} \in C_{0}^{\infty}\left(\mathbb{R}^{n}\right)$ and for $n / \delta<q<n$ and $p, 1 / p=1 / s-1 / n,\left\|A_{j, \varepsilon}-A_{j}\right\|_{L^{p}},\left\|B_{k, \varepsilon}-B_{k}\right\|_{L^{p}}$, $\left\|A_{j, \varepsilon}-A_{j}\right\|_{L^{q}}$ and $\left\|B_{k, \varepsilon}-B_{k}\right\|_{L^{q}}$ converge to zero as $\varepsilon \rightarrow 0$. Therefore by the remark following Lemma 3.3, $\left\|\mathscr{A}_{j, \varepsilon} G_{0}(z) \mathscr{B}_{k, \varepsilon}-\mathscr{A}_{j} G_{0}(z) \mathscr{B}_{k}\right\| \rightarrow 0$ as $\varepsilon \rightarrow 0$. Hence we may assume $\quad A_{j}, \quad B_{k} \in C_{0}^{\infty}\left(\mathbb{R}^{n}\right)$. By $\quad(3.42) \quad$ and $(3.43), \quad T_{v_{k}}^{*} \mathscr{A}_{j} G_{0}(z) \mathscr{B}_{k} T_{v_{k}}$ 
$=T_{v_{k}}^{*} \mathscr{A}_{j} T_{v_{k}} G_{0}(z)\left(I \otimes B_{j}\right)$. Since $I \otimes B_{j}$ is the multiplication by $B_{j}(x)$ and $T_{v k}^{*} \mathscr{A}_{j} T_{v_{k}}$ is the multiplication by $\left.A_{j}\left(x-v_{j}-v_{k}\right) t\right), v_{j}-v_{k} \neq 0$, we get the desired result.

(ii) Let $f(\tau) \in C^{\infty}\left(\mathbb{R}^{1}\right)$ be such that $f(\tau)=1$ for $|\tau| \geqq 2 f(\tau)=0$ for $|\tau| \leqq 1$ and $|f(\tau)|$ $\leqq 1$ for $1 \leqq|\tau| \leqq 2$. Let $F_{R}$ be the operator defined as $F_{R}=\mathscr{F}_{t}{ }^{-1} f(\tau / R) \mathscr{F}_{t}$.

$$
\begin{aligned}
& A(x-v t) G_{0}( \pm i) B(x)=A(x-v t) G_{0}( \pm i) F_{R} B(x) \\
& \quad+A(x-v t) G_{0}( \pm i)\left(1-F_{R}\right) B(x) .
\end{aligned}
$$

We first prove $A(x-v t) G_{0}( \pm i)\left(1-F_{R}\right) B(x) \in \mathbf{B}_{\infty}\left(K_{2}\right)$ for any $R>0$. Let $E_{ \pm}(\tau, \xi)$ $=(1-f(\tau / R))\left(\tau+|\xi|^{2} / 2 \pm i\right)^{-1}$. Obviously

$$
\begin{aligned}
& G_{0}( \pm i)\left(1-F_{R}\right)=F_{(t, x)}^{-1} F_{ \pm}(\tau, \xi) F_{(t, x)} \\
& \left|(\partial / \partial \tau)^{\alpha}(\partial / \partial \xi)^{\beta} E_{ \pm}(\tau, \xi)\right| \leqq C_{R, \alpha, \beta}^{\gamma}(1+|\tau|)^{-\gamma}(1+|\xi|)^{-2},
\end{aligned}
$$

for any multi-index $\alpha$ and $\beta$, and any $\gamma \geqq 0$. Hence $G_{0}( \pm i)\left(1-F_{R}\right) \in \mathbf{B}\left(L^{2}\left(\mathbb{R}^{n+1}\right)\right.$, $\left.H^{2}\left(\mathbb{R}^{n+1}\right)\right)$ and by Corollary 3.11

$$
A(x-v t) G_{0}( \pm i)\left(1-F_{R}\right) B(x) \in \mathbf{B}\left(L^{2}\left(\mathbb{R}^{n+1}\right), H^{2}\left(\mathbb{R}^{n+1}\right)\right) .
$$

On the other hand by Lemma 3.10, (3.67), (3.68) and the obvious inequality

$$
(1+|x-v t|)^{-\rho}(1+|x|)^{-\rho} \leqq C_{\rho}(1+|x|+|t|)^{-\rho},
$$

we get

$$
A(x-v t) G_{0}( \pm i)\left(1-F_{R}\right) B(x) \in \mathbf{B}\left(L^{2}\left(\mathbb{R}^{n+1}\right), L_{\rho}^{2}\left(\mathbb{R}^{n+1}\right)\right),
$$

for any $\rho>0$. Hence by Lemma 3.9, $A(x-v t) G_{0}( \pm i)\left(1-F_{R}\right) B(x)$ is a compact operator on $L^{2}\left(\mathbb{R}^{n+1}\right)=\Omega_{2}$.

(iii) Finally we prove

$$
\lim _{R \rightarrow \infty}\left\|A(x-v t) G_{0}( \pm i) F_{R} B(x)\right\|=0,
$$

which completes the proof of Lemma 3.8. Since $A, B \in C_{0}^{\infty}\left(\mathbb{R}^{n}\right)$, $A(x-v t) \mathscr{F}_{(t, x)}^{-1} \in \mathbf{B}\left(L^{2}\left(\mathbb{R}^{n+1}\right)\right)$ and $\mathscr{F}_{(t, x)} B(x) \in \mathbf{B}\left(L^{2}\left(\mathbb{R}^{n+1}\right), L^{2}\left(\mathbb{R}^{1}\right) \otimes H^{k}\left(\mathbb{R}^{n}\right)\right)$ for any $k \geqq 0$. Therefore for proving (3.72), it suffices to prove that the multiplication operator by the function $f(\tau / R)\left(\tau+\xi^{2} / 2 \pm i\right)^{-1}$ regarded as an operator from $L^{2}\left(\mathbb{R}^{1}\right) \otimes H^{k}\left(\mathbb{R}^{n}\right)$ to $L^{2}\left(\mathbb{R}^{n+1}\right)$ converges to zero in operator norm as $R \rightarrow \infty$, for some $k>0$. We take $k=1$. Then by Sobolev's embedding theorem (see Kuroda [10], p. 4.13, Theorem 1 and p. 4.26, Theorem $\left.1^{\prime}\right)$, we have for $u \in H^{1}\left(\mathbb{R}^{n}\right)$,

$$
\sup _{\rho \geqq 0} \rho^{(n-1) / 2}\|u(\rho \cdot)\|_{L^{2}\left(S^{n-1}\right)} \leqq C\|u\|_{H^{1}\left(\mathbb{R}^{n}\right)},
$$

where $S^{n-1}$ is the unit sphere in $\mathbb{R}^{n}$. Let $N<R$. Then

$$
\begin{aligned}
& \int \frac{|f(\tau / R) u(\tau, \xi)|^{2}}{\left|\tau+\xi^{2} / 2 \pm i\right|^{2}} d \tau d \xi \\
& \leqq \int_{\substack{\left|\tau+\xi^{2} / 2\right| \leqq N \\
|\tau| \geqq R}}+\int_{\substack{\left|\tau+\xi^{2} / 2\right| \geqq N \\
|\tau| \leqq R}} \frac{|u(\tau, \xi)|^{2}}{\left(\tau+\xi^{2} / 2\right)^{2}+1} d \tau d \xi
\end{aligned}
$$




$$
\begin{aligned}
& \leqq \\
& \int_{\sqrt{2 \tau-2 N} \leqq|\xi| \leqq \sqrt{2 \tau+2 N}}|u(-\tau, \xi)|^{2} d \tau d \xi+\left(N^{2}+1\right)^{-1}\|u\|^{2} \\
& =\int_{R}^{\infty} d \tau\left\{\int_{\sqrt{2 \tau-2 N}}^{\sqrt{2 \tau+2 N}} d \rho\left(\rho^{n-1} \int_{s^{n-1}}|u(-\tau, \rho \omega)|^{2} d \omega\right)\right\}+\left(N^{2}+1\right)^{-1}\|u\|^{2} \\
& \leqq C \int_{R}^{\infty} d \tau \int_{\sqrt{2 \tau-2 N}}^{\sqrt{2 \tau+2 N}}\|u(-\tau, \cdot)\|_{H^{1}\left(\mathbb{R}^{n}\right)}^{2} d \rho+\left(N^{2}+1\right)^{-1}\|u\|^{2} \\
& \leqq C \int_{R}^{\infty}\left(\sqrt{2 \tau+2 N}-\sqrt{2 \tau-2 N)}\|u(-\tau, \cdot)\|_{H^{1}\left(\mathbb{R}^{n}\right)}^{2} d \rho+\left(N^{2}+1\right)^{-1}\|u\|^{2}\right. \\
& \leqq C \sqrt{2 N}(R-N)^{-1 / 2} \int_{-\infty}^{\infty}\|u(\tau, \cdot)\|_{H^{1}\left(\mathbb{R}^{n}\right)}^{2} d \tau+\left(N^{2}+1\right)^{-1}\|u\|^{2} \\
& \leqq\left(C \sqrt{2 N}(R-N)^{-1 / 2}+\left(N^{2}+1\right)^{-1}\right)\|u\|_{L^{2}\left(\mathbb{R}^{1}\right) \otimes H^{1}\left(\mathbb{R}^{n}\right)}^{2} .
\end{aligned}
$$

Here we used (3.73) in the fourth step. Deviding both sides of (3.74) by $\|u\|_{L^{2}\left(\mathbb{R}^{1}\right) \otimes H^{1}\left(\mathbb{R}^{n}\right)}^{2}$ and letting $R \rightarrow \infty$ first and then $N \rightarrow \infty$, we get the desired result easily. This completes the proof of Lemma 3.8.

(Q.E.D.)

Lemma 3.12 For any $j=1,2, \ldots, N$ and $z \in \mathbb{C}_{ \pm} \cup \mathbb{R}^{1}, 1+\mathscr{A}_{j} G_{0}(z) \mathscr{B}_{j}^{*}$ has an inverse in $\mathbf{B}\left(\Re_{2}\right)$. Moreover $\left(1+\mathscr{A}_{j} G_{0}(z) \mathscr{B}_{j}^{*}\right)^{-1}$ is uniformly bounded and Hölder continuous in $z \in \mathbb{C}_{ \pm} \cup \mathbb{R}^{1}$.

Proof. By (3.42) and (3.43),

$$
\begin{aligned}
1+ & \mathscr{A}_{j} G_{0}(z) \mathscr{B}_{j}^{*}=T_{v_{j}}\left(1+\left(1 \otimes A_{j}\right)\left(-i \partial / \partial t \otimes I+I \otimes H_{0}-z\right)^{-1}\left(I \otimes B_{j}\right)\right) T_{v_{j}}^{*} \\
& =T_{v_{j}} \mathscr{F}_{t}^{-1}\left(1+\left(I \otimes A_{j}\right)\left(\tau+I \otimes H_{0}-z\right)^{-1}\left(I \otimes B_{j}\right)\right) \mathscr{F}_{t} T_{v_{j}}^{*},
\end{aligned}
$$

where $\tau$ is the conjugate variable of $t$. Now we regard as $L^{2}\left(\mathbb{R}^{1}\right) \otimes L^{2}\left(\mathbb{R}^{n}\right)=$ $\int^{\oplus} L^{2}\left(\mathbb{R}^{n}\right) d \tau$; we write as $Q_{j}(z)=A_{j}\left(H_{0}-z\right)^{-1} B_{j}$. Then by (3.75),

$$
1+\mathscr{A}_{j} G_{0}(z) \mathscr{B}_{j}^{*}=T_{v_{j}} \mathscr{F}_{t}{ }_{t}^{-1}\left(\int^{\oplus}\left(1+Q_{j}(z-\tau)\right) d \tau\right) \mathscr{F}_{t} T_{v_{j}}^{*} .
$$

Since $\left(1+Q_{j}(z)\right)^{-1}$ exists for $z \in \mathbb{C}_{ \pm} \cup \mathbb{R}^{1}$ and is uniformly bounded and Hölder continuous there by Sect. 1, (C). (i) and Assumption (III), (3.76) implies that

$$
\left(1+\mathscr{A}_{j} G_{0}(z) \mathscr{B}_{j}^{*}\right)^{-1}=T_{v_{j}} \mathscr{F}_{t}^{-1}\left(\int\left(1+Q_{j}(z-\tau)\right)^{-1} d \tau\right) \mathscr{F}_{t} T_{v_{j}}^{*}
$$

exists and is uniformly bounded and Hölder continuous in $z \in \mathbb{C}_{ \pm} \cup \mathbb{R}^{1}$.

Lemma 3.13. Let $j \neq k, j, k=1,2, \ldots, N$. Then

(1) $\Gamma_{j} T_{v_{j}}^{*} \mathscr{B}_{k} \in \mathbf{B}\left(L^{2}\left(\mathbb{R}^{n+1}\right), L_{\delta}^{2}\left(\mathbb{R}^{1}\right)\right)$;

(2) $\mathscr{A}_{j} T_{v_{k}} \Gamma_{k}^{*}, \mathscr{B}_{j} T_{v_{k}} \Gamma_{k}^{*} \in \mathbf{B}\left(L_{\rho}^{2}\left(\mathbb{R}^{1}\right), L_{\rho+\delta}^{2}\left(\mathbb{R}^{n+1}\right)\right), \rho \leqq 0$; 
(3) $\Gamma_{j} T_{v_{j}}^{*} \mathscr{B}_{k}^{*} \mathscr{A}_{k} T_{v_{k}} \Gamma_{k}^{*}$ is a multiplication operator by a function $f_{j, k}(t)$ satisfying $\left|f_{j, k}(t)\right| \leqq C\left(1+t^{2}\right)^{-\delta}$.

Proof. We frequently use the following two results:

(i) If $v \neq v^{\prime}$ and $\rho \leqq 0$,

$$
\left(1+|x-v t|^{2}\right)^{\rho}\left(1+\left|x-v^{\prime} t\right|^{2}\right)^{\rho} \leqq C\left(1+|x|^{2}+|t|^{2}\right)^{\rho}
$$

where $C$ is a constant depending only on $\rho$ and $v-v^{\prime}$;

(ii) By (1.6) and Sobolev's embedding theorem, for any $0 \leqq \rho$, $\left(1+|x|^{2}\right)^{\rho} \phi_{j}(x) \in L^{q}\left(\mathbb{R}^{n}\right)$, where $q=2 n /(n-4)$ if $n \geqq 5 ; 1 \leqq q<\infty$ is arbitrary if $n=4$; and $1 \leqq q \leqq \infty$ is arbitrary if $n=3$. Therefore by Hölder's inequality,

$$
\left(1+|x|^{2}\right)^{\delta} \phi_{j}(x) \in L^{2 n /(n-2)}\left(\mathbb{R}^{n}\right), \quad n \geqq 3 .
$$

(1) $\left|\left(\Gamma_{j} T_{v_{j}}^{*} \mathscr{B}_{k} u\right)(t)\right| \leqq \int\left|\phi_{j}(x)\left(1+\left|x-\left(v_{k}-v_{j}\right) t\right|^{2}\right)^{-\delta / 2} u\left(x-v_{j} t, t\right)\right| d x$.

By Schwartz's inequality and (3.78), the R.H.S. is majorized by the square root of

$$
C\left(1+t^{2}\right)^{-\delta}\left(\int\left(1+x^{2}\right)^{\delta}\left|\phi_{j}(x)\right|^{2} d x\right)\left(\int|u(x, t)|^{2} d x\right)
$$

Hence $\quad \int\left(1+t^{2}\right)^{-\delta}\left|\left(\Gamma_{j} T_{v_{j}}^{*} \mathscr{B}_{k} u\right)(t)\right|^{2} d t \leqq C\left\|\left(1+x^{2}\right)^{\delta / 2} \phi_{j}\right\|^{2}\|\| u \|^{2}$.

(2) $\left|\left(\mathscr{A}_{j} T_{v_{k}} \Gamma_{k}^{*} \sigma\right)(t, x)\right|=\left|A_{j}\left(x-v_{j} t\right) \phi_{k}\left(x-v_{k} t\right) \sigma(t)\right|$.

Hence by Remark 1.1, (1.6), (3.78), (3.79) and Hölder's inequality, we have

$$
\begin{aligned}
& \int\left(1+x^{2}+t^{2}\right)^{\rho+\delta}\left|\left(\mathscr{A}_{j} T_{v_{k}} \Gamma_{k}^{*} \sigma\right)(t, x)\right|^{2} d x d t \\
& \leqq C \int\left(1+x^{2}+t^{2}\right)^{\rho}\left|W_{j, 1}\left(x-v_{j} t\right)+W_{j, 2}\left(x-v_{j} t\right)\right|^{2} \\
& \cdot\left(1+\left|x-v_{k} t\right|^{2}\right)^{\delta}\left|\phi_{k}\left(x-v_{k} t\right)\right|^{2}|\sigma(t)|^{2} d x d t \\
& \leqq C\left(\left\|W_{j, 1}\right\|_{L^{n}}^{2}\left\|\left(1+x^{2}\right)^{\delta} \phi_{k}\right\|_{L^{2 n /(n-2)}}^{2}+\left\|W_{j, 2}\right\|_{L^{\infty}}^{2}\left\|\left(1+x^{2}\right)^{\delta} \phi_{k}\right\|_{L^{2}}^{2}\right)\|\sigma\|_{L_{\rho}^{2}}^{2} .
\end{aligned}
$$

The other is easier to prove and the proof is omitted.

(3) $\Gamma_{j} T_{v_{j}}^{*} \mathscr{A}_{k} \mathscr{B}_{k}^{*} T_{v_{k}} \Gamma_{k}^{*}$ is obviously a multiplication operator by a function $f_{j, k}(t)$ which is majorized by

$$
\int\left|\phi_{j}(x) V_{k}\left(x-\left(v_{k}-v_{j}\right) t\right) \phi_{k}\left(x-v_{k} t\right)\right| d x .
$$

By Hölder's inequality, (3.78) and (3.79), (3.80) is majorized by constant times $\left\|\left(1+x^{2}\right)^{\delta} \phi_{j}\right\|_{L^{2 n /(n-2)}}\left\|\left(1+x^{2}\right)^{\delta} \phi_{k}\right\|_{L^{2 n /(n-2)}}\left\|V_{k}\right\|_{L^{n / 2}}\left(1+t^{2}\right)^{-\delta}$.

(Q.E.D.)

Lemma 3.14. For any $j, k=1,2, \ldots, N$,

$$
\begin{aligned}
& \mathscr{B}_{j}^{-1} T_{v_{j}} \Gamma_{j}^{*} \in \mathbf{B}\left(L^{2}\left(\mathbb{R}^{1}\right), \quad L^{2}\left(\mathbb{R}^{n+1}\right)\right) ; \\
& \mathscr{A}_{j} T_{v_{j}} \Gamma_{j}^{*} \Gamma_{j} T_{v_{j}}^{*} \mathscr{B}_{j}^{-1}, \quad \mathscr{B}_{k}^{-1} T_{v_{j}} \Gamma_{j}^{*} \Gamma_{j} T_{v_{j}}^{*} \mathscr{B}_{k} \in \mathbf{B}\left(L^{2}\left(\mathbb{R}^{n+1}\right)\right) .
\end{aligned}
$$

Proof. (3.81) is obvious by (1.6). We prove (3.82). By Schwartz's inequality, $f_{j}(t, x)=\left(\mathscr{A}_{j} T_{v_{j}} \Gamma_{j}^{*} \Gamma_{j} T_{v_{j}}^{*} \mathscr{B}_{j}^{-1} u\right)(t, x) \quad$ satisfies $\quad\left|f_{j}(t, x)\right|=\mid A_{j}\left(x-v_{j} t\right) \phi_{j}\left(x-v_{j} t\right)$ $\cdot\left\|\phi_{j}(x)\left(1+x^{2}\right)^{\delta / 2}\right\|\|u(t, \cdot)\|$. Hence by Hölder's inequality, $\left\|f_{j}\right\| \leqq\left\|\left(1+x^{2}\right)^{\delta / 2} \phi_{j}\right\|$ - $\left\|\phi_{j}\right\|_{L^{2 n /(n-2)}}\left\|A_{j}\right\|_{L^{n}}\|u\|$. We prove the second. The case $j=k$ is obvious by (3.18). We assume $j \neq k$. By Lemma 3.13, (1), it suffices to prove 
$\mathscr{B}_{k}^{-1} T_{v_{j}} \Gamma_{j}^{*} \in \mathbf{B}\left(L_{\delta}^{2}\left(\mathbb{R}^{1}\right), \quad L^{2}\left(\mathbb{R}^{n+1}\right)\right) . \quad$ By triangle inequality $\quad\left(1+t^{2}\right)^{-\delta / 2}(1+$ $\left.|x-v t|^{2}\right)^{\delta / 2} \leqq C(1+x)^{\delta / 2}$. Hence

$$
\int\left|\left(\mathscr{B}_{k}^{-1} T_{v_{j}} \Gamma_{j}^{*} \sigma\right)(t, x)\right|^{2} d t d x \leqq C\left\|\left(1+x^{2}\right)^{\delta / 2} \phi_{j}\right\|^{2}\left\|\left(1+t^{2}\right)^{\delta / 2} \sigma\right\|^{2},
$$

from which the desired result follows.

Lemma 3.15. For any $j, k=1,2, \ldots, N, \mathscr{M}_{j, k}(z)=\mathscr{A}_{j} \mathscr{G}_{j}^{c}(z) \mathscr{B}_{k}$ satisfies the following properties.

(1) $\mathscr{M}_{j, k}(z)$ is a $\mathbf{B}\left(\Omega_{2}\right)$-valued analytic function of $z \in \mathbb{C}_{ \pm}$and is uniformly bounded there.

(2) $\mathscr{M}_{j, k}(z)$ can be extended to the closed cut plane $\mathbb{C}_{ \pm} \cup \mathbb{R}^{1}$ as a $\mathbf{B}\left(\Re_{2}\right)$-valued Hölder continuous function of $z$.

(3) If $j \neq k, \mathscr{M}_{j, k}(z)$ is a compact operator on $\mathfrak{R}_{2}$ for any $z \in \mathbb{C}_{ \pm} \cup \mathbb{R}^{1}$.

(4) $\left\|\mathscr{M}_{j, k}(z)\right\| \rightarrow 0$ as $|\operatorname{Im} z| \rightarrow \infty$.

Proof. By the resolvent equation

$$
\begin{aligned}
& \left(-i \partial / \partial t \otimes I+I \otimes H_{j}-z\right)^{-1} \\
& \quad=G_{0}(z)-G_{0}(z)\left(I \otimes V_{j}\right)\left(-i \partial / \partial t \otimes I+I \otimes H_{j}-z\right)^{-1} .
\end{aligned}
$$

Multiply to the both sides of (3.84), $T_{v_{j}} A_{j}$ from the left and $\left(I \otimes P_{j}^{c}\right) T_{v_{j}}^{*} \mathscr{B}_{k}$ from the right. Then by (3.42), (3.43) and (3.46), we get

$$
\mathscr{M}_{j, k}(z)=\mathscr{A}_{j} G_{0}(z) T_{v_{j}}\left(I \otimes P_{j}^{c}\right) T_{v_{j}}^{*} \mathscr{B}_{k}-\mathscr{A}_{j} G_{0}(z) \mathscr{B}_{j} \mathscr{M}_{j, k}(z) .
$$

Therefore by Lemma 3.12,

$$
\mathscr{M}_{j, k}(z)=\left(1+\mathscr{A}_{j} G_{0}(z) \mathscr{B}_{j}\right)^{-1} \mathscr{A}_{j} G_{0}(z) T_{v_{j}}\left(I \otimes P_{j}^{c}\right) T_{v_{j}}^{*} \mathscr{B}_{k} .
$$

Here $\mathscr{A}_{j} G_{0}(z) T_{v_{j}}\left(I \otimes P_{j}^{c}\right) T_{v_{j}}^{*} \mathscr{B}_{k}=\mathscr{A}_{j} G_{0}(z) \mathscr{B}_{k}-\mathscr{A}_{j} G_{0}(z) \mathscr{B}_{k} \mathscr{B}_{k}^{-1} T_{v_{j}} \Gamma_{j}^{*} \Gamma_{j} T_{v_{j}}^{*} \mathscr{B}_{k}$. Since $\mathscr{A}_{j} G_{0}(z) \mathscr{B}_{k}$ satisfies Lemma 3.3 and is compact if $j \neq k$ by Lemma 3.8; $\mathscr{B}_{k}^{-} T_{v_{j}} \Gamma_{j}^{*} \Gamma_{j} T_{v_{j}}^{*} \mathscr{B}_{k} \in \mathbf{B}\left(\mathscr{R}_{2}\right)$ by (3.82) and $\left(1+\mathscr{A}_{j} G_{0}(z) \mathscr{B}_{j}\right)^{-1}$ satisfies Lemma 3.12, $\mathscr{M}_{j, k}(z)$ satisfies all the properties of Lemma 3.15.

We set as

(Q.E.D.)

$$
\mathfrak{Y}=\bigoplus_{j=1}^{N}\left(L^{2}\left(\mathbb{R}^{n+1}\right) \oplus L_{\delta}^{2}\left(\mathbb{R}^{1}\right)\right)
$$

with natural Hilbert space structure.

Lemma 3.16. (1) $L(z)$ is a $\mathbf{B}\left(\mathfrak{X}_{2}, \mathfrak{Y}\right)$-valued analytic function of $z \in \mathbb{C}_{ \pm}$and is uniformly bounded there.

(2) $L(z)$ can be extended to $\mathbb{C}_{ \pm} \cup \mathbb{R}^{1}$ as a $\mathbf{B}\left(\mathfrak{X}_{2}, \mathfrak{Y}\right)$-valued Hölder continuous function.

Proof. Lemma 3.13,(1) implies that $\Gamma_{j} T_{v_{j}}^{*} \in \mathbf{B}\left(\mathfrak{X}_{2}, L_{\delta}^{2}\left(\mathbb{B}^{1}\right)\right)$. Hence applying Lemma 3.15 to $\mathscr{A}_{j} \mathscr{G}_{j}^{c}(z)$, we get easily the statements of the lemma.

(Q.E.D.)

Finally we prove the following lemma.

Lemma 3.17. The operator valued function $A(z)$ satisfies the following properties: 
(1) $A(z)$ is a $\mathbf{B}(\mathfrak{Y})$-valued analytic function of $z \in \mathbb{C}_{ \pm}$and is uniformly bounded there.

(2) $A(z)$ can be extended to $\mathbb{C}_{ \pm} \cup \mathbb{R}^{1}$ as a $\mathbf{B}(\mathfrak{Y})$-valued Hölder continuous function of $z$.

(3) For any $z \in \mathbb{C}_{ \pm} \cup \mathbb{R}^{1}, A^{2}(z)$ is a compact operator on $\mathfrak{Y}$.

(4) $\lim _{|\operatorname{Im} z| \rightarrow \infty}\left\|A^{2}(z)\right\|=0$.

Corollary 3.18. There exists a closed null set $e \subset \mathbb{R}^{1}$ such that $(1+A(z))^{-1}$ can be extended to $\mathbb{C}_{ \pm} \cup\left(\mathbb{R}^{1} \backslash e\right)$ as a $\mathbf{B}(\mathfrak{Y})$-valued locally Hölder continuous function of $z$.

Proof. 1) $\mathscr{A}_{j} \mathscr{G}_{j}^{c}(z) \mathscr{B}_{k}$ satisfies the statments of Lemma 3.15 .

2) $\Gamma_{j} T_{v_{j}}^{*} \mathscr{B}_{k}$ satisfies Lemma $3.13,(1)$.

3) Put $C_{j, k}(z)=\Gamma_{j} T_{v_{j}}^{*} \mathscr{B}_{k}^{*} \mathscr{A}_{k} T_{v_{k}} \Gamma_{k}^{*} G_{k}(z)$. Since $\Gamma_{j} T_{v_{j}}^{*} \mathscr{B}_{k}^{*} \mathscr{A}_{k}^{*} T_{v_{k}} \Gamma_{k}^{*}$ is a multiplication operator by $f_{j, k}(t)$ satisfying the estimate $\left|f_{j, k}(t)\right| \leqq C\left(1+t^{2}\right)^{-\delta}$ by Lemma 3.13, (3), Lemma 3.6 implies that $C_{j, k}(z)$ is a $\mathbf{B}_{\infty}\left(L_{\delta}^{2}\left(\mathbb{R}^{1}\right)\right)$-valued bounded analytic function of $z \in \mathbb{C}_{ \pm}$and can be extended to $\mathbb{C}_{ \pm} \cup \mathbb{R}^{1}$ as a Hölder continuous function; $\left\|C_{j, k}(z)\right\| \rightarrow 0$ as $|\operatorname{Im} z| \rightarrow \infty$.

4) Put $\mathscr{N}_{j, k}(z)=\mathscr{A}_{j} \mathscr{G}_{j}^{c}(z) \mathscr{B}_{k}^{*} \mathscr{A}_{k} T_{v_{k}} \Gamma_{k}^{*} G_{k}(z)$. We show that $\mathscr{N}_{j, k}(z)$ is a $\mathbf{B}_{\infty}\left(L_{\delta}^{2}\left(\mathbb{R}^{1}\right), L^{2}\left(\mathbb{R}^{n+1}\right)\right)$-valued bounded analytic function of $z \in \mathbb{C}_{ \pm}$and can be extended to $\mathbb{C}_{ \pm} \subset \mathbb{R}^{1}$ as a Hölder continuous function; $\left\|\mathscr{N}_{j, k}(z)\right\| \rightarrow 0$ as $|\operatorname{Im} z| \rightarrow \infty$ and is uniformly bounded. Using (3.85) for $\mathscr{A}_{j} G_{j}^{c}(z) \mathscr{B}_{k}$ we get

$$
\begin{aligned}
\mathscr{N}_{j, k}(z)= & \mathscr{A}_{j} G_{0}(z) T_{v_{j}}\left(I \otimes P_{j}^{c}\right) T_{v_{j}}^{*} \mathscr{B}_{k}^{*} \mathscr{A}_{k} T_{v_{k}} \Gamma_{k}^{*} G_{k}(z) \\
& -\mathscr{A}_{j} G_{0}(z) \mathscr{B}_{j} \mathscr{N}_{j, k}(z) .
\end{aligned}
$$

Therefore by Lemma 3.12, we have

$$
\begin{aligned}
& \mathscr{N}_{j, k}(z)=\left(1+\mathscr{A}_{j} G_{0}(z) \mathscr{B}_{j}\right)^{-1} \mathscr{A}_{j} G_{0}(z) T_{v_{j}}\left(I \otimes P_{j}^{c}\right) T_{v_{j}}^{*} \mathscr{B}_{k}^{*} \mathscr{A}_{k} T_{v_{k}} \Gamma_{k}^{*} G_{k}(z) . \\
& \quad=\left(1+\mathscr{A}_{j} G_{0}(z) \mathscr{B}_{j}\right)^{-1} \mathscr{A}_{j} G_{0}(z) \mathscr{B}_{k}^{*}\left(1-\mathscr{B}_{k}^{-1} T_{v_{j}} \Gamma_{j}^{*} \Gamma_{j} T_{v_{j}}^{*} \mathscr{B}_{k}^{*}\right) \mathscr{A}_{k} T_{v_{k}} \Gamma_{k}^{*} G_{k}(z) .
\end{aligned}
$$

Now we apply Lemma 3.12 to the first factor; Lemma 3.15 to $\mathscr{A}_{j} G_{0}(z) \mathscr{B}_{k}^{*}$; Lemma $3.14,(8.82)$ to $\left(1-\mathscr{B}_{k}^{-1} T_{v_{j}} \Gamma_{j}^{*} \Gamma_{j} T_{v_{j}}^{*} \mathscr{B}_{k}^{*}\right)$; Lemma 3.6 and Lemma 3.13, (2) to $\mathscr{A}_{k} T_{v_{k}} \Gamma_{k}^{*} G_{k}(z)$. Then we get the desired result.

Combining these results (1)-(4), we get easily the statements of Lemma 3.17. Corollary 3.18 is a well-known result of Lemma 3.17 .

(Q.E.D.)

\subsection{Completion of the Proof of the Theorem}

What is left to be proved is $R(\mathscr{W})=\mathfrak{\Re}_{2}$. We take $e \subset \mathbb{R}^{1}$ as in Corollary 3.18. We set $I=\mathbb{R}^{1} \backslash e ; \Omega_{1}$ and $\Omega_{2}$ as (3.1); $K_{1}$ and $K_{2}$ as (3.3),(3.4) and (3.5); $J$ as (3.2); $Y(z)$ as (3.60); $\mathfrak{X}_{1}$ and $\mathfrak{X}_{2}$ as (3.63). We first check that all the assumptions of Theorem 3.2 are satisfied. Condition (1) is satisfied by Lemma 3.1. In condition (2), for $j=1,2, \mathfrak{X}_{j}$ is a dense linear submanifold of $\Omega_{j}$ and is a Hilbert space. (2.i) is satisfied by Lemma 3.7. The equation (3.15) is satisfied by (3.59). $Y(z)$ is a $\mathbf{B}\left(\mathfrak{X}_{2}, \mathfrak{X}_{1}\right)$-valued strongly continuous function of $z \in \mathbb{C}_{ \pm}(I) \cup I$, since $(1+A(z))^{-1} L(z)$ is a $\mathbf{B}\left(\mathfrak{X}_{2}, \mathfrak{Y}\right)$-valued strongly continuous function of $z \in \mathbb{C}_{ \pm}(I) \cup I$ by Lemma 3.16 and Corollary 3.18; the injection operator from $\mathfrak{X}_{2}$ into $R(\mathscr{B})^{*}$ is bounded and $T_{v_{j}} \Gamma_{j}^{*}$ satisfies (3.81). Hence 
the all assumptions are satisfied. Therefore by Theorem 3.2. we see that $K_{1}$ and $K_{2}$ are absolutely continuous on $I ; R(\mathscr{W}) \supset E_{2}(I) \Re_{2}$. Let us admit the following lemma for a moment.

Lemma 3.19. $K_{2}$ is spectrally absolutely continuous on $\boldsymbol{\Omega}_{2}$.

By Lemma 3.19, $E_{2}(I) \Re_{2}=\Re_{2}$ since $\mathbb{R}^{1} \backslash I$ has Lebesgue measure zero. Thus $R(\mathscr{W})$ $=\mathfrak{R}_{2}$. This completes the proof of the theorem.

Proof of Lemma 3.19. Let us define a one parameter unitary group $I(\tau)$, $-\infty<\tau<\infty$, and a unitary operator $T$ on $\mathfrak{R}_{2}$ as

$$
\begin{aligned}
& (I(\tau) u)(t, x)=u(t-\tau, x), \\
& (T u)(t, \cdot)=U(t, 0) u(t, \cdot), \quad u \in \Omega_{2} .
\end{aligned}
$$

By Sect. 1, (B), (ii), (3.4) and (3.5), we can easily see that

$$
\exp \left(-i \tau K_{2}\right)=T I(\tau) T^{*} \text {. }
$$

Since $I(\tau)$ has the absolutely continuous generator $-i(\partial / \partial t),(3.92)$ obviously implies that $K_{2}$ is absolutely continuous.

(Q.E.D.)

Acknowledgement. This work was initiated while the author was visiting the University of Virginia. He is indebted to J. S. Howland and I. Herbst for stimulating discussions at the early stage of the work.

\section{References}

1. Agmon, S.: Spectral properties of Schrödinger operators and scattering theory, Ann. Scuola Norm. Pisa, Ser. IV, 2.2, 151-218 (1975)

2. Combes, J. M.: Relatively compact interactions in many particle systems. Commun. Math. Phys. 12, 283-295 (1969)

3. Faddeev, L. D.: Mathematical aspect of the three body problem in the quantum mechanical scattering theory. Israel program for scientific translations, Jerusalem, 1965 (English translation from Russian)

4. Ginibre, J., Moulin, M.: Hilbert space approach to the quantum mechanical three body problem. Ann. Inst. H. Poincaré 21, 97-145 (1974)

5. Howland, J.: Stationary scattering theory for time-dependent Hamiltonians. Math. Ann. 207, 315-335 (1974)

6. Howland, J.: Abstract stationary theory for multi-channel scattering theory. J. Funct. Anal. 22, 250-282 (1976)

7. Kato, T.: Wave operators and similarity for some non-selfadjoint operators. Math. Ann. 162, 258-279 (1966)

8. Kato, T.: Two space scattering theory, with applications to many body problems. J. Fac. Sci. Univ. Tokyo, Sec. IA 24, 503-514 (1977)

9. Konno, R., Kuroda, S. T.: On the finiteness of perturbed eigenvalues. J. Fac. Sci. Univ. Tokyo, Sec. IA 8, 55-63 (1966)

10. Kuroda, S. T. : An introduction to scattering theory. Aarhus University Lecture Note, 1978

11. Reed, M., Simon B.: Method of modern mathematical physics, Vol. II. Fourier analysis and selfadjointness. New York: Academic Press 1975

12. Reed, M., Simon, B.: Method of modern mathematical physics, Vol. III. Scattering theory. New York: Academic Press 1978

13. Reed, M. Simon, B. Method of modern mathematical physics, Vol. IV. Analysis of operators. New York: Academic Press 1978 
14. Simon, B.: Quantum mechanics for Hamiltonians defined as quadratic forms. Princeton, NJ Princeton Univ. Press 1971

15. Stein, E. M.: Singular integrals and differentiability properties of functions. Princeton, NJ: Princeton Univ. Press 1970

16. Yajima, K.: Scattering theory for Schröedinger equations with potentials periodic in time. J. Math. Soc. Jpn 29, 729-743 (1977)

17. Yajima, K.: An abstract stationary approach to three body scattering. J. Fac. Sci. Univ. Tokyo, Sec. IA 25, 109-132 (1978)

18. Yosida, K.: Functional analysis. Berlin, Heidelberg, New York: Springer 1968

19. Lions, L. J., Magenes, E.: Non-homogeneous boundary value problems and applications, I. Berlin, Heidelberg, New York: Springer 1972

Communicated by B. Simon

Received September 6, 1979 